\title{
Nonlinear effects in time-resolved spectra of DAVs
}

\author{
J. Ising and D. Koester
}

\author{
Institut für Theoretische Physik und Astrophysik, Universität Kiel, 24098 Kiel, Germany \\ e-mail: koester@astrophysik.uni-kiel.de
}

Received 26 June 2000 / Accepted 2 April 2001

\begin{abstract}
Numerical simulations of light curves of variable DA white dwarfs (ZZ Ceti stars) predict flux amplitudes with surface distributions different from the spherical harmonics of the pulsation mode in deeper layers. In contrast to the results of the perturbation analysis by Goldreich and $\mathrm{Wu}$, this is also true for the fundamental period of the flux variation. As a consequence, normalized amplitude spectra depend not only on the mode number $l$ but also on pulsation amplitude and inclination. Another new result is that with increasing amplitude of the pressure variation below the convection zone, the flux variation at the surface goes through a maximum and then decreases again.
\end{abstract}

Key words. stars: white dwarfs - stars: atmospheres - stars: interiors - convection - stars: variable: general

\section{Introduction}

The ZZ Ceti stars are the coolest class of pulsating white dwarfs. They are multi-periodic $g$-mode pulsators with periods in the range of $100 \mathrm{~s}$ to $1000 \mathrm{~s}$. In contrast to the PG 1159 objects and the variable DB only a few modes are observed in each individual ZZ Ceti star. The number of observed pulsation periods, the amplitudes and the shape of the light curves of these stars change systematically within the instability strip (see e.g. Clemens 1993). At the hot (blue) edge, small amplitude pulsations are observed with sinusoidal light curves. The cooler ZZ Ceti stars show more modes with large amplitudes of more than $10 \%$ with overtones and additional frequencies, formed by sums and differences of the fundamental frequencies of the modes.

The small number of unstable modes forms an obstacle to standard mode identification techniques (identifying the "quantum numbers" $l$ and $m$ of the spherical harmonics of a mode), which rely mostly on periods and period spacings of unstable frequencies in the power spectrum. In recent years, time-resolved spectroscopy of white dwarfs has become possible and was used to identify the spherical harmonics of the modes. This mode identification technique uses the wavelength dependence of normalized pulsation amplitudes and takes advantage of the wavelength dependence of the limb darkening and different cancellation of the flux variation for different spherical harmonics (Robinson et al. 1982; Brassard et al. 1995; Robinson et al. 1995; Kepler et al. 2000; Clemens \& Kerkwijk 2000). This

Send offprint requests to: J. Ising, e-mail: ising@astrophysik.uni-kiel.de diagnostic technique depends on the assumption that the flux amplitude on the visible surface varies with the spherical harmonic $(l, m)$ of the mode.

This assumption - which we will call "linear" - is subject to criticism, at least for larger amplitudes. For large amplitudes, non-sinusoidal light curves are observed, in contrast to the sinusoidal light curves for small amplitudes. Because the ZZ Ceti stars are non-radial pulsators, the amplitude varies over the surface of the star and one would expect a variation of the shape of the light curves over the surface for large amplitudes as well. This is in contradiction to the assumption of a flux variation with spherical harmonics. For a quantitative description of the integrated light curve and spectra we will need a theoretical understanding of the reaction of the local surface flux to an imposed pressure variation at deeper layers. This theory should demonstrate how the surface variation becomes increasingly non-sinusoidal with increasing amplitude of the pressure variation.

Such a description was provided by Brickhill (1983), who proposed that the thin convection zone below the surface of the ZZ Ceti stars has an important effect on the light curves. Brickhill showed, in a series of papers, that numerical simulations of the light curves can qualitatively reproduce the observed flux variations. This result was confirmed by $\mathrm{Wu}(1998)$ and $\mathrm{Wu}(2000)$.

Goldreich \& Wu (1999, 1999a) and Wu \& Goldreich (1998a) studied the driving mechanism of the ZZ Ceti stars in a linear perturbation analysis. Their work lead to impressive progress in the understanding of the driving of the pulsation modes, but it their linear analysis they consider only the entropy change in a convection zone of 
constant depth. Wu $(1998,2000)$ extended this work to take into account the depth changes, but used only static models of convection zone.

The numerical integrations we have performed for this paper show that for larger amplitudes the depth varies significantly during one cycle. At each time step during the pulsation the structure remains different from any static structure, leading to modifications of the nonlinear effects, which appear already in the perturbation analysis using static structures, as described in this paper.

We extend Brickhill's work and show that the convection zone strongly influences the spatial distribution of surface amplitudes, which for larger amplitudes cannot anymore be described by spherical harmonics. This cannot be neglected in the technique of mode identification by time-resolved spectroscopy. In the next section we describe the model used for our numerical calculations. First we will introduce the relatively simple system of ordinary differential equations to solve the time-dependent structure of the envelope of ZZ Ceti stars, obtained by allowing only a restricted time dependence of the basic equations. To examine the validity of this restriction for large amplitude pulsation, we introduce the full time-dependent equations in the following subsection. The boundary conditions have a large influence on the results of the numerical simulations; the following subsection is devoted to this topic. Section 3 describes the results of a light curve simulation for a plane-parallel column with a fixed pulsation amplitude. In Sect. 4 we consider that the pulsation amplitude varies over the surface for non-radial pulsators like the ZZ Ceti stars, and describe the surface distribution of the flux for different maximum amplitudes and spherical harmonics. The technique used to calculate the total flux together with the expected consequences for the timedependent spectroscopy are discussed in Sect. 5 Finally we give our main conclusion in Sect. 6 .

\section{The numerical model}

Within her analysis, Wu $(1998,2000)$ calculates the local response of the surface flux to a sinusoidal variation of pressure and flux in a layer at the bottom of the convection zone. Due to the change in extent and structure of the convection zone its reaction to the perturbation of the input flux is nonlinear, leading to the appearance of higher harmonics in the surface flux (Wu 2000).

The main result is that the amplitude of the fundamental period depends linearly and that of the first overtone quadratically on the amplitude below the convection zone. The phase delays between surface flux and pressure variation are constant, independent of amplitude. This important result guarantees that the spatial distribution over the stellar surface for the fundamental period is still given by the same spherical harmonic, which describes the variation in the deeper layers. This justifies the usual assumptions made in the analysis of time-dependent spectroscopy.
The starting point of our own study is the numerical model of Brickhill. In this model the convective layer is considered as a plane-parallel column. The lower boundary of this column lies below the surface convection zone, the upper boundary is directly below the photosphere. The sinusoidal relative pressure variation is assumed to be constant through the whole column, an assumption justified in the work by Brickhill and Goldreich and Wu.

In the simulation we calculate the transfer of energy and the time-dependent temperature structure numerically in a way similar to Brickhill's work. Some improvements over his work are state-of-the-art equation of state from Saumon et al. (1995) and OPAL opacity data (Iglesias \& Rogers 1996), and our own model atmosphere grids, which are described in Finley et al. (1997).

In this paper we want to show the principal effects for the analysis of time-resolved spectroscopy. Since we are not aiming at a detailed comparison with observations, it is sufficient to investigate only one equilibrium model with one effective temperature and one pulsation period. We take a stellar model with $T_{\text {eff }}=11350 \mathrm{~K}$ and $\log g=8$ and a pulsation period of $100 \mathrm{~s}$. We use only one parameter for the mixing length theory (ML2/ $\alpha=0.6$ ); the thermal relaxation time scale is $4.5 \mathrm{~s}$. With this choice our model is representative for the conditions near the blue edge of the instability strip. All our calculations and results of this study are based on this set of parameters.

\subsection{The basic equations}

The main aim of the model calculation is to determine the temperature structure as a function of time. The temperature disturbance $\delta T$ for a static model can be calculated from

$\delta T=\frac{\delta Q}{C_{\mathrm{p}} \Delta M}+\nabla_{\mathrm{ad}} T \frac{\delta P}{P}$

Equation (1) is equivalent to the first law of thermodynamics (e.g. Cox 1980, p. 37) and can be found in Brickhill (1983) as well. The first term on the right describes the non-adiabatic heating by the additional heat energy $\delta Q$ of a mass $\Delta M$ with the heat capacity $C_{\mathrm{p}}$. The second term is the adiabatic contribution to the temperature change due to the relative pressure variation $\frac{\delta P}{P}$ in an environment with an adiabatic temperature gradient $\nabla_{\text {ad }}$.

To calculate the heat energy $\delta Q$ we consider a finite volume element with the cross section $A$ and a height $\Delta z$. Introducing a discretization and finite volume elements at this point, we can avoid the use of partial differential equations. In a plane-parallel symmetry, the flux has only a vertical component. If $\Delta F$ is the difference between the outgoing and the incoming flux for a mass element, the heat energy accumulated in a time interval $\Delta t$ is given by

$\delta Q=\int_{0}^{\Delta t} \mathrm{~d} t A \Delta F$

or written in differential form

$\frac{\mathrm{d} \delta Q}{\mathrm{~d} t}=A \Delta F$. 
To calculate the mass $\Delta M$ of the volume element we follow the procedure of Brickhill. We take the pressure of the non-pulsating star in hydrostatic equilibrium to define the vertical depth scale. Following Brickhill we introduce $\tilde{\omega} \equiv \ln P$ as the independent variable of the problem. The mass element for a fixed pressure difference $\Delta \tilde{\omega}$ can be written as

$$
\begin{aligned}
\Delta M & =A \int_{z_{1}}^{z_{2}} \mathrm{~d} z \varrho(t) \\
& =A \int_{\tilde{\omega}_{1}+\Delta \tilde{\omega}}^{\tilde{\omega}_{1}} \mathrm{~d} \tilde{\omega} \varrho(t) \frac{\mathrm{d} z}{\mathrm{~d} \tilde{\omega}}=A \int_{\tilde{\omega}_{1}}^{\tilde{\omega}_{1}+\Delta \tilde{\omega}} \mathrm{d} \tilde{\omega} \frac{P(t)}{g} .
\end{aligned}
$$

As Brickhill assumed, and was confirmed by Goldreich \& $\mathrm{Wu}$ (1999), the relative pressure variation can be taken as constant through the whole column. We further assume a constant surface gravity $g$ throughout the column. This simplifies the expression for the mass element:

$$
\begin{aligned}
\Delta M & =A \frac{\exp \left(\frac{\delta P}{P}(t)\right)}{g} \exp (\tilde{\omega}) \int_{0}^{\Delta \tilde{\omega}} \mathrm{d} \tilde{\omega}^{\prime} \exp \left(\tilde{\omega}^{\prime}\right) \\
& =A \frac{P(t)}{g} \hat{f}(\Delta \tilde{\omega})
\end{aligned}
$$

with

$\hat{f}(x) \equiv \exp (x)-1$.

In a first order approach we can combine Eqs. (1), (3) and (4), by taking the derivative with respect to the time of (1). In this first order approach we are only concerned with the time-dependency of $\delta T, \delta Q$ and $\frac{\delta P}{P}$. We get

$$
\frac{\mathrm{d} T}{\mathrm{~d} t}=\frac{\Delta F g}{C_{\mathrm{p}} P \hat{f}(\Delta \tilde{\omega})}+\nabla_{\mathrm{ad}} T \frac{\mathrm{d}}{\mathrm{d} t} \frac{\delta P}{P} .
$$

For all the mass elements of the vertical column, Eq. (6) defines a system of ordinary differential equations (ODE) for the temperature structure. The fluxes describe the coupling of the equations at each grid-point of the column. In our approximation we calculate the flux locally from the actual temperatures and temperature gradients. It is given by

$$
F=F_{\mathrm{r}}+F_{\mathrm{c}},
$$

where the radiative flux $F_{\mathrm{r}}$ can be calculated with the diffusion approximation and the convective flux $F_{\mathrm{c}}$ by means of the mixing length theory. We use a time-independent formulation of the mixing length theory and assume an instantaneous adjustment of the total flux to the temperature and pressure structure of the column. To check the validity of this assumption we have also used the timedependent Kuhfuss model for convection (Kuhfuss 1986; Wuchterl \& Feuchtinger 1998) and found no differences between the time-dependent and the time-independent model calculations. This result holds strictly, if the kinetic energy of the convection does not contribute to the thermal energy, but the differences are also small if we take such a coupling into account.
The rapid variation of the flux with the temperature leads to short relaxation time scales near the upper boundary of the column. This leads to a stiff behavior of the ODE. Consequently we use a Gear solver (Gear 1971) to integrate Eq. (6).

\subsection{The full time-dependent equations}

The differential formulation of (1) leads in principle to reliable solutions for large perturbations as well. But to obtain Eq. (6) we have ignored all time-dependencies except for $\delta T, \delta Q$ and $\frac{\delta P}{P}$. For large amplitudes $\left(\frac{\delta P}{P}>5 \%\right)$, this cannot be justified any longer, since temperature and specific heat capacity can change by large amounts. In a first step to correct for this we can replace all quantities in Eq. (6) by their current values instead of those of the equilibrium model. Since this may not be sufficient for short pulsation periods ( $100 \mathrm{~s}$ ), we have modified Eq. (6) and taken into account all terms produced by the derivative of (1) with respect to time

$\left[1+x_{1}-x_{2}\right] \frac{\mathrm{d} T}{\mathrm{~d} t}=\frac{\Delta F g}{C_{\mathrm{p}} P \hat{f}(\Delta \tilde{\omega})}+\nabla_{\mathrm{ad}} T \frac{\mathrm{d}}{\mathrm{d} t} \frac{\delta P}{P}-x_{3}+x_{4} \cdot(8)$

In the following all thermodynamic quantities are functions of $T$ and $P$, and a partial derivative with respect to $T$ means that $P$ is to be kept constant and vice versa. The correction terms $x_{1}$ and $x_{3}$ due to the non-adiabatic term of Eq. (1) are

$$
\begin{aligned}
& x_{1}=\frac{\delta Q}{C_{\mathrm{p}}^{2} \Delta M} \frac{\partial C_{\mathrm{p}}}{\partial T} \\
& x_{3}=\frac{\delta Q}{C_{\mathrm{p}}^{2} \Delta M} \frac{\partial C_{\mathrm{p}}}{\partial P} \frac{\partial P}{\partial t} .
\end{aligned}
$$

The correction terms $x_{2}$ and $x_{4}$ are produced by the derivative of the adiabatic term of (1)

$$
\begin{aligned}
& x_{2}=\left(\frac{\partial \nabla_{\mathrm{ad}}}{\partial T} T+\nabla_{\mathrm{ad}}\right) \frac{\delta P}{P} \\
& x_{4}=\frac{\partial \nabla_{\mathrm{ad}}}{\partial P} T \frac{\delta P}{P} \frac{\partial P}{\partial t} .
\end{aligned}
$$

The derivative of the mass element with respect to the time is calculated again under the assumption, that the relative pressure perturbation is constant through the whole column. Then this variation is inversely related to the change of the horizontal area

$\frac{\delta A}{A}=-\frac{\delta P}{P}$

This leads to

$\frac{\mathrm{d} \Delta M}{\mathrm{~d} t}=\left(\frac{\mathrm{d} A}{\mathrm{~d} t} P(t)+A(t) \frac{\mathrm{d} P}{\mathrm{~d} t}\right) \frac{\hat{f}(\Delta \tilde{\omega})}{g}=0$.

In Eq. (8) appear both the time-derivative of $\delta Q$ and $\delta Q$ itself. This changes the structure of the equations from ordinary differential equations to a set of integro-differential equations. We solve this system by a splitting procedure. 
In the inner step, we solve the differential equations for a time step short compared to the pulsation period with constant $\delta Q$. After this time step, $\delta Q$ is updated. For time steps of about $0.25 \%$ of the period, the achieved accuracy is high enough, since the integral only appears in higher order terms.

The contributions of $x_{1}$ and $x_{2}$ are important for large pulsation amplitudes, caused by the large and rapid changing of the temperature, whereas all terms including a derivative of $\nabla_{\text {ad }}$ are unimportant. Table 1 compares the amplitudes of the fundamental and the first overtone of the photospheric flux variation as a result of (6) with (8) for our model with a period of $100 \mathrm{~s}$. For (6) we take the current values of all quantities, instead of the values of the equilibrium model.

Table 1. Fourier amplitudes for the photospheric flux variation. Columns 2 and 4 display the amplitude of the fundamental for Eq. (6) (ODE) and Eq. (8) (IDE), respectively. Columns 3 and 5 show the amplitudes of the first overtone. All quantities are functions of the pressure amplitude $\frac{\delta P}{P}$.

\begin{tabular}{|c|c|c|c|c|}
\hline & \multicolumn{2}{|c|}{ ODE } & \multicolumn{2}{c|}{ IDE } \\
\hline$\frac{\delta P}{P}$ & $A_{1}[\%]$ & $A_{2}[\%]$ & $A_{1}[\%]$ & $A_{2}[\%]$ \\
\hline 0.02 & 1.71 & 0.24 & 1.71 & 0.25 \\
0.04 & 3.64 & 1.17 & 3.65 & 1.23 \\
0.06 & 5.14 & 2.01 & 5.18 & 2.13 \\
0.08 & 6.17 & 2.61 & 6.29 & 2.83 \\
0.10 & 6.78 & 2.95 & 7.04 & 3.32 \\
0.12 & 6.97 & 2.97 & 7.45 & 3.56 \\
0.14 & 6.74 & 2.67 & 7.54 & 3.54 \\
0.16 & 6.09 & 2.01 & 7.31 & 3.25 \\
0.18 & 5.17 & 1.17 & 6.78 & 2.68 \\
\hline
\end{tabular}

The differences for large pressure amplitudes are significant in the solutions of (6) and (8), but the qualitative behavior is the same in both cases. Since the numerical effort is not very much larger for solving the IDE, this method will be implemented in future versions of our code. The ODE solution (6) is adequate for a qualitative discussion and all results given in this paper are based on (6) with current values for all quantities. One should remember, however, that the results for large amplitudes - though qualitatively correct - may be affected by this approximation.

\subsection{Boundary conditions}

The basic system of ODE (6) looks like an initial value problem, but this is only partially correct. To obtain Eq. (2) we have introduced a discretization of the column, to replace the spatial derivatives of the flux with finite differences. The mathematical structure of a partial differential equation survives in the coupling of the ODEs and the necessity to define boundary conditions for the flux.
For the lower boundary we assume the adiabatic approximation. The matter in the region below the convection zone is well approximated as a completely ionized hydrogen plasma. Under this assumption the flux perturbation is given by (see Goldreich \& Wu 1999)

$\frac{\delta F}{F}=\frac{3-3 \kappa_{\varrho}-2 \kappa_{\mathrm{T}}}{5} \frac{\delta P}{P}$,

where $\kappa_{\varrho}$ and $\kappa_{\mathrm{T}}$ are the logarithmic derivatives of the opacity with respect to the density and temperature. This adiabatic assumption is the weakest point of the approximations in the numerical model. In order to test this assumption and also to find the optimum location of the lower boundary we have made a numerical experiment.
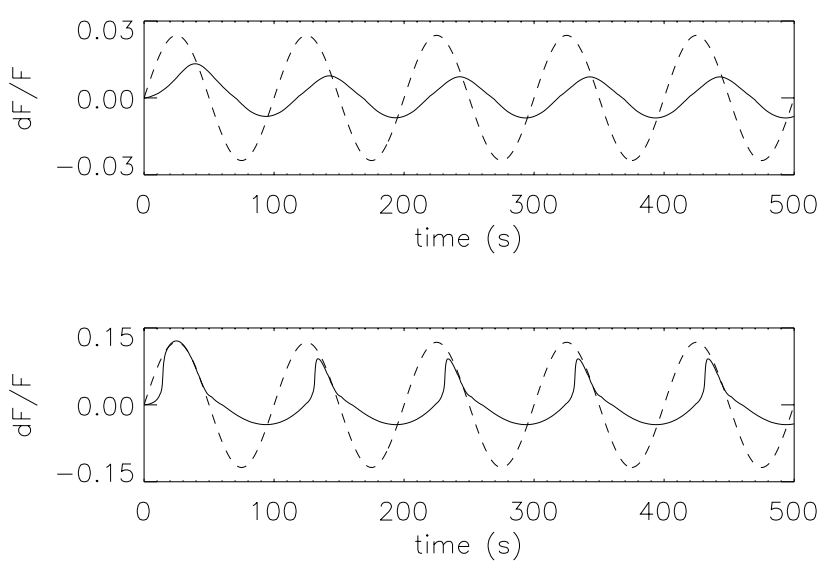

Fig. 1. The variation of the photospheric flux (solid line) and the flux variation at the lower boundary (dashed line) are plotted for a $1 \%$ (upper panel) and a $5 \%$ pressure amplitude (lower panel).

We assume a column with a lower boundary much deeper than the bottom of the convection zone with a constant pressure amplitude in the whole column. The depth of the lower boundary is limited by the growing computing costs, because a pulsation simulation has to be done for at least one thermal time scale of the whole column to reach a stationary situation. For our model we choose a thermal time scale of $1500 \mathrm{~s}$ and a value of $\tilde{\omega}=22$ to fix the lower boundary for this experiment. At this lower boundary we apply a sinusoidal flux perturbation and study the resulting flux at a point clearly below the maximum extent of the convection zone, but far away from the lower boundary. At this point the flux is no longer sinusoidal but shows a fundamental with amplitude $A_{1}$ and a first overtone with amplitude $A_{2}$. The phases are shifted relative to the bottom flux by $\phi_{1}$ and $\phi_{2}$ respectively, where a positive phase means that the variation is lagging behind the perturbation. One should note that these effects would be absent in a completely radiative star; they are caused by the delayed reaction of the convection zone, which determines the outer boundary for the envelope structure.

Table 2 shows the results at the point with $\tilde{\omega}=19.45$. The first overtone at this point remains at least one order of magnitude smaller than the fundamental amplitude 
and the phase shift between pressure and flux variation remains small. We follow Brickhill in our conclusion that the adiabatic flux variation at the lower boundary is a good approximation if the propagation zone of the $g$-modes is far away from the convective layer. This is true for short period pulsations as considered in our model, which represents the "blue edge" of the instability strip. For our pulsation simulations we then fixed our lower boundary at $\tilde{\omega}=20$.

Table 2. Influence of non-adiabatic terms at the lower boundary: results of the experiment described in the text. The 2nd column displays the amplitude of the sinusoidal flux variation at the lower boundary. The 3rd and 4th column is the amplitude and phase of the fundamental at the point $\tilde{\omega}=19.45$, which is always below the convection zone. The first overtones are shown in Cols. 5 and 6.

\begin{tabular}{|c|c|c|c|c|c|}
\hline$\frac{\delta P}{P}$ & $\left(\frac{\delta F}{F}\right)_{\mathrm{lb}}[\%]$ & $A_{1}[\%]$ & $\phi_{1}\left[^{\circ}\right]$ & $A_{2}[\%]$ & $\phi_{2}\left[^{\circ}\right]$ \\
\hline 0.02 & 4.40 & 4.72 & 2.67 & 0.05 & -98.43 \\
0.04 & 8.80 & 9.43 & 2.67 & 0.19 & -97.35 \\
0.06 & 13.20 & 14.09 & 2.84 & 0.40 & -97.80 \\
0.08 & 17.60 & 18.62 & 3.12 & 0.69 & -97.80 \\
0.10 & 22.00 & 23.05 & 3.43 & 1.08 & -97.52 \\
0.12 & 26.40 & 27.39 & 3.75 & 1.56 & -97.35 \\
0.14 & 30.80 & 31.59 & 4.09 & 2.10 & -97.92 \\
0.16 & 35.20 & 35.61 & 4.47 & 2.72 & -99.01 \\
0.18 & 39.60 & 39.45 & 4.88 & 3.44 & -100.32 \\
\hline
\end{tabular}

To find an upper boundary condition we make the assumption, that the photosphere reaches the static structure for each time step instantaneously. As discussed e.g. by Brickhill (1983) this approximation is valid for small thermal time scales compared with the pulsation period. We choose the upper boundary at the pressure point with the optical depth $\approx 10$, where this condition is clearly fulfilled. The advantage to take the optical depth clearly greater than 1 at the upper boundary of the column is, that the time-dependent calculations can be done with the diffusion approximation for the radiative flux. The determination of the photospheric structure requires a detailed radiative transfer calculation, but without consideration of terms with time-derivatives. To connect these two different calculations we assume, that the combination of the actual flux, pressure and temperature is the same at the upper boundary of the column as in one specific static photospheric model. To find the flux we use a grid of static models and find the known combination of pressure and temperature in this grid for each time step separately and interpolate the flux from these models. This procedure avoids a further linearization at the upper boundary, but is in principle equivalent to Brickhill's method.

\section{Numerical results for a column}

In this section we study the reaction of the convection zone and the shape and amplitude of the surface flux variation for a column on the stellar surface.

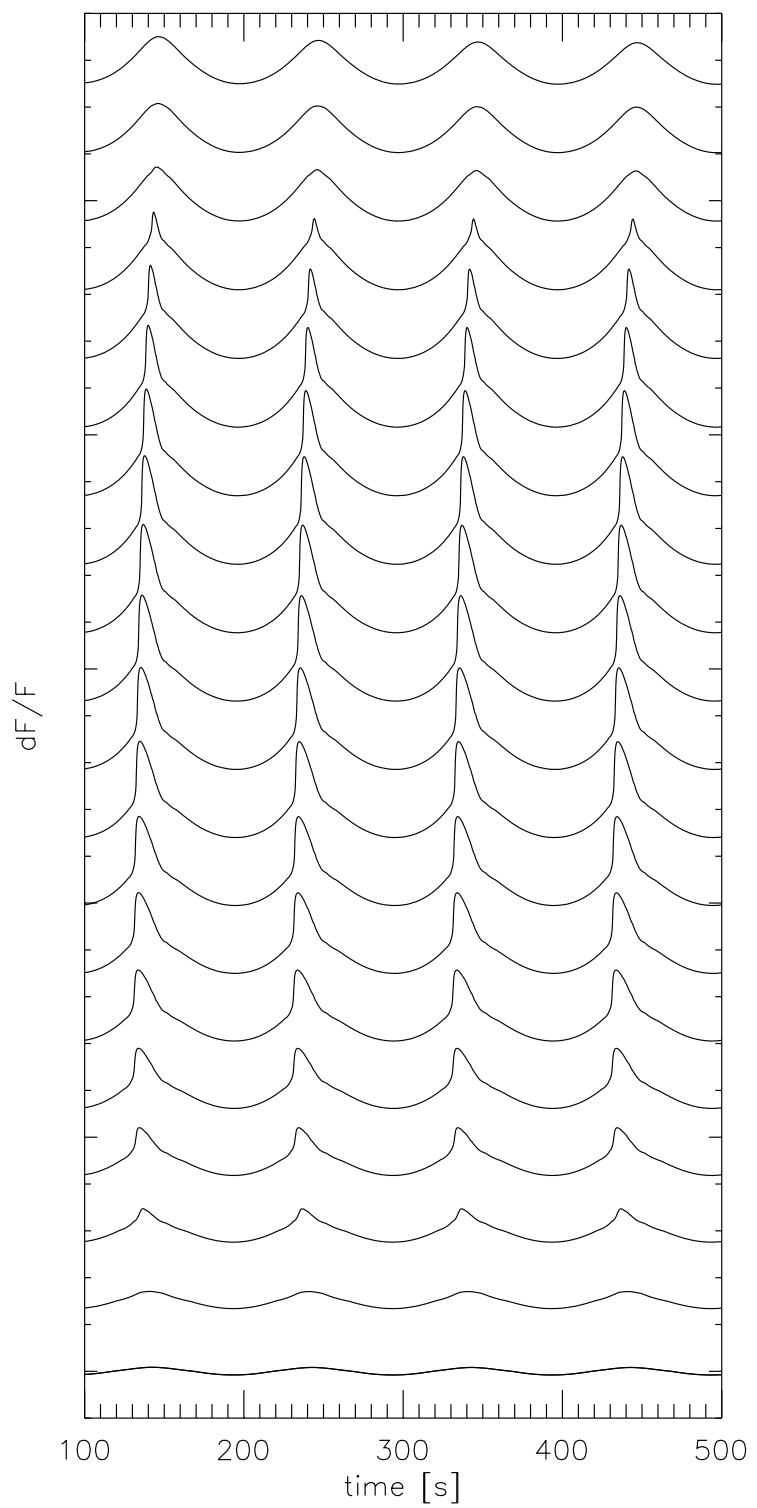

Fig. 2. Shape of the flux variation for different pressure amplitudes. The bottom line is the light curve for $1 \%$ amplitude, the increment between curves is $1 \%$. The light curve at the top of the panel is for $20 \%$ pressure amplitude.

For small amplitude pulsations with $1 \%$ pressure variation (see upper panel in Fig. 1), the photospheric flux (solid line) is shifted in phase relative to the flux of the adiabatic layers (dashed line) and the amplitude is reduced. As shown by Brickhill (1983), the phase shift and the reduced amplitude is caused by the delay in the heat transfer due to the necessary adjustment of the convective layers to a change in the input flux. If the flux is increased, the depth of the convective layer is shrinking as required by a static envelope solution corresponding to a higher effective temperature. A large amount of heat is necessary to change convective into radiative layers and to increase the heat content of the remaining convection zone, which has to be supplied by the input flux. Consequently, the photospheric flux follows the input flux with a delay of the 


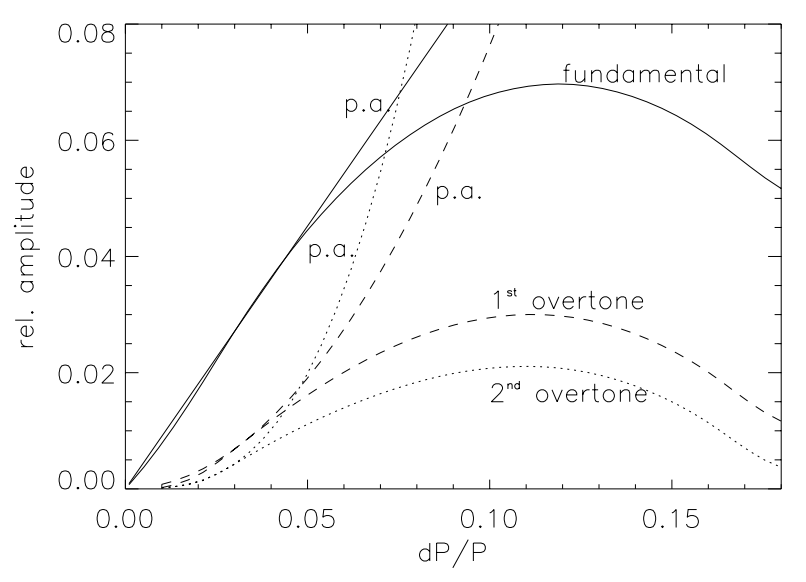

Fig. 3. Amplitudes of the Fourier coefficients relative to the mean flux for a local column. The numerical results are labeled with the name of the harmonic. The curves marked with p.a. indicate the linear and quadratic relations predicted by the perturbation analysis.

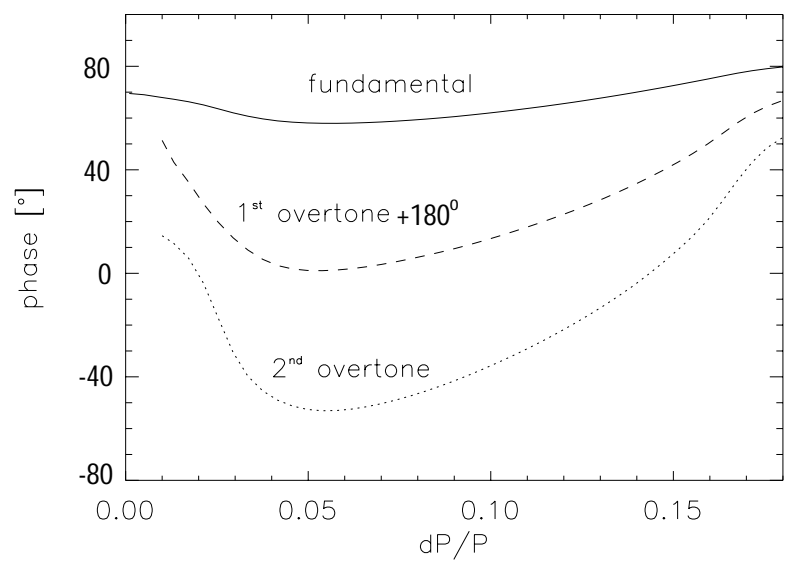

Fig. 4. Phases of the fundamental and overtones relative to the pressure variation in a local column for the numerical results. The linear perturbation analysis predicts constant phases with values which depend on the equilibrium model.

order of the thermal time scale of the convective layer. If the input flux varies periodically with a period comparable to the thermal time scale, this delay results in a phase shift and an amplitude reduction.

Figure 2 summarizes this variation of the light curves with changing pressure amplitudes.

For small amplitudes the variation of the surface flux remains sinusoidal and the amplitude increases linearly with the pressure amplitude as predicted by the perturbation analysis.

For larger amplitudes, e.g. 5\% (lower panel Fig. 1) the extent of the the convective zone varies significantly during the pulsation cycle, and the stratification may even become completely radiative shortly after maximum compression. At that instant the photospheric flux follows directly the adiabatic flux variation, causing the steep increase and sharp maxima in the light curves.

Increasing the pressure amplitudes even further (20\%), the surface flux variation becomes sinusoidal again, with

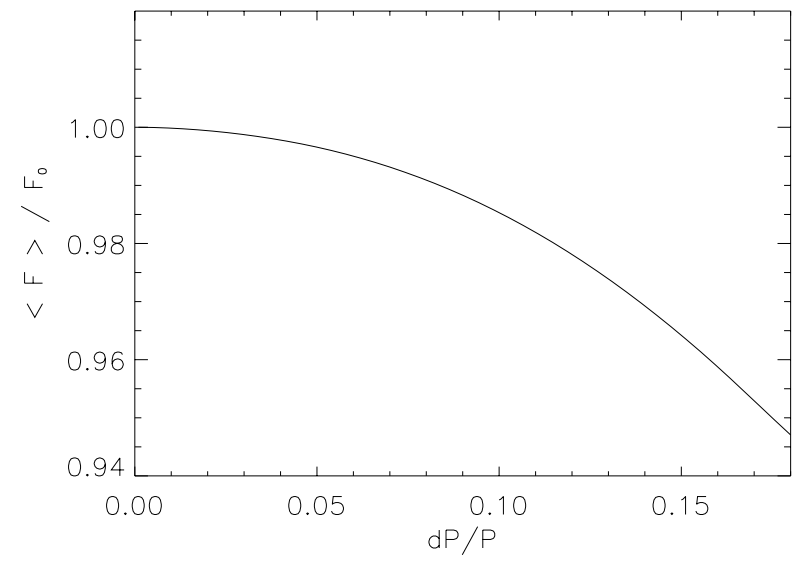

Fig. 5. Time-averaged flux relative to the flux below the convection zone.

small amplitudes. In this case, a large fraction of the heat flux is transformed into mechanical energy, and the remaining average flux corresponds to a model with lower (time-averaged) effective temperature and thicker convection zone. The thermal time constant of this convection zone becomes large compared to the pulsation period, resulting in a strong reduction of the flux amplitude.

We emphasize that this conversion of heat to mechanical energy in the column (which remains qualitatively the same with the higher numerical accuracy of the IDE method) does not directly tell us anything about the global excitation or damping of the mode. Our calculation is based on energy arguments in an open system: the enforced variations of the pressure and column cross section carry away any difference of incoming and outgoing heat flux. In order to really study the excitation of the mode, one would need to calculate the behavior of the mode throughout the whole star. It is quite possible that the increase of the mechanical energy in the outer envelope is more than balanced by a damping in the interior; it is also possible that the energy goes into the excitation of other modes. This problem will be studied further in future simulations, which include the study of the $P \mathrm{~d} V$ term in Eq. (1).

The sharp maxima in the light curves imply the appearance of higher harmonics in the Fourier decomposition. Figure 3 demonstrates, how the relative amplitudes of fundamental, first, and second overtone increase with increasing pressure amplitude. For low amplitudes, the results confirm the predictions of the perturbation analysis (linear and quadratic dependence for fundamental and first overtone); for amplitudes larger than 5\%, however, strong deviations become apparent.

The sign and even the magnitude of the phase shifts in Fig. 4 approximately agree with the predictions of the perturbation analysis: the fundamental for the flux is lagging behind the pressure, whereas the in the first harmonic it is leading. However, quite unexpectedly from the predictions of Goldreich and $\mathrm{Wu}$, for very small amplitudes the phases do not become constant. The shift demonstrates 
the change of the relative contributions from the compression in the convection zone, which is strictly in phase, and the flux variation below the convection zone, which is phase shifted during the propagation through the convective layer. While at very low amplitudes the numerical result may be questionable, we are confident that this is not the case for pressure amplitudes larger than about $1 \%$. This result needs further study, and we will attempt in a future paper to reconcile the results of numerical and perturbational analysis.

Another difference to the perturbation analysis of Goldreich and $\mathrm{Wu}$ is the dependence of the mean local flux on the pressure amplitude (see Fig. 5). The flux from below the convection zone is reduced by a fraction of energy that is converted into mechanical energy to drive the pulsation mode. This fraction grows quadratically with the pressure amplitude. Consequently the mean flux is reduced very efficiently for large amplitudes.

\section{Surface distribution}

In the preceding sections we have discussed the local reaction of the photospheric flux to an assumed fixed pressure amplitude. In this section we consider the whole surface of the star, assuming that the spatial structure of the pressure perturbation as well as the flux perturbation below the convection zone is described by a spherical harmonic of mode numbers $l$ and $m$.

For small amplitudes - because of the linear dependence of surface amplitudes on pressure amplitudes - the distribution of the surface flux amplitude follows directly the pressure and is therefore described by the same spherical harmonic. But this is only true if one ignores the small phase changes of the flux variation. Due to this phase shift the maximum flux amplitude is reached at a later time for smaller amplitudes. This effect leads to a deviation of the photospheric flux distribution from the spherical harmonic of the mode, but the flux amplitude at the surface is still a monotonous function of the pressure amplitude. This changes with increasing amplitude: because of the nonmonotonous local relation the maximum flux amplitude at the surface does not coincide with the maximum of the pressure amplitude and e.g. for $m=0$ modes the maximum flux amplitude is not reached at the poles. Since also the phases change with amplitude, different latitudes on the surface reach the maximum flux at different times.

Figures 6 to 8 show the surface distribution for $l=$ $1,2, m=0$ modes as three-dimensional plot with graylevel indicating the effective temperature, and in graphical form the dependence on polar angle $\mu=\cos \vartheta$. Shown are three different cases for small, medium, and large pressure amplitudes.

For $l=2$ modes the change due to the nonlinear relation between pressure amplitude and surface flux amplitude may be quite dramatic: whereas for small amplitudes the maximum amplitudes are at the poles, with a smaller relative maximum at the equator, the situation can be completely reversed at large amplitudes (see Fig. 8).
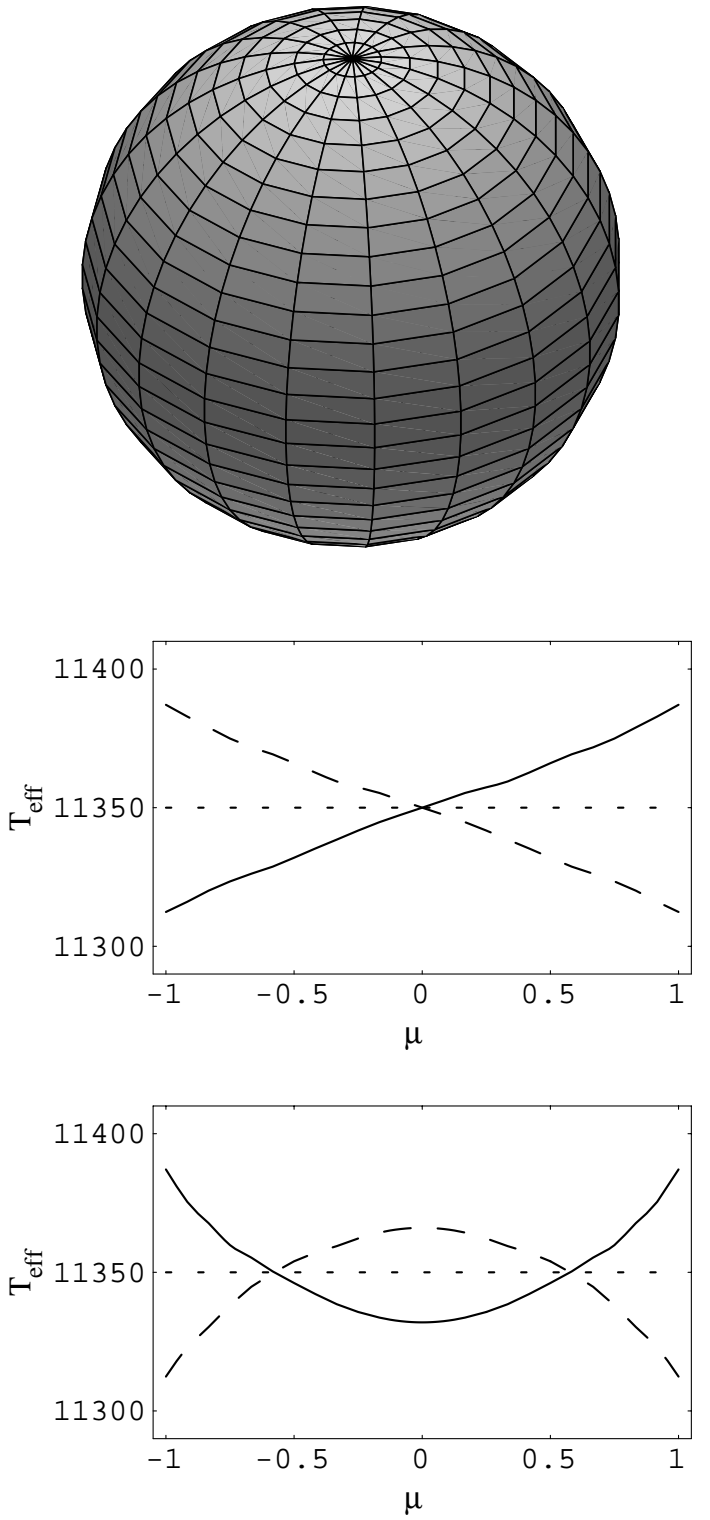

Fig. 6. Surface distribution of the effective temperature near the maximum of the integrated flux. The gray-level of the plot (top, for an $l=2$ mode) is normalized to the maximum $T_{\text {eff }}$ (white) and minimum $T_{\text {eff }}$ (black) on the surface. The middle panel shows in graphical form the temperature distribution with angle $\mu=\cos \vartheta$ for $l=1$ at three different times, the bottom panel the same but for $l=2$. The maximum pressure amplitude is $2 \%$ (small amplitude).

\section{Flux integration and time-resolved spectra}

From these results it follows that the surface distribution of the flux variation in general has a much more complicated form than is assumed traditionally (meaning spherical harmonics describing all variations on the stellar surface). In this section we want to answer two questions: First, we want to find an optimal strategy to calculate the wavelength dependent amplitude spectra, and second, we want to discuss the major effects on time-resolved spectra one has to expect, if the linear assumption is no longer appropriate. 

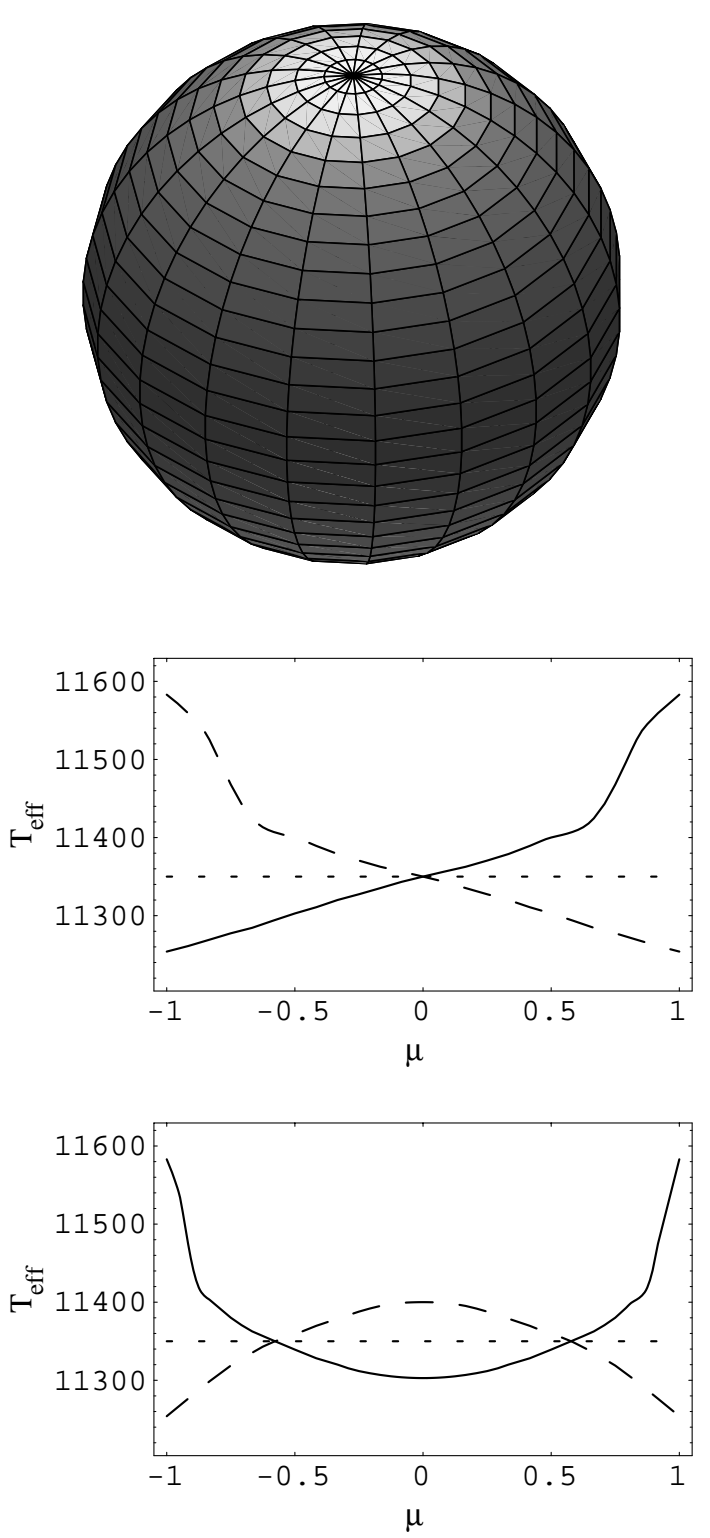

Fig. 7. Similar to Fig. 6, but for a maximum pressure amplitude of $5 \%$.

The general problem is to find the time-dependent flux $\mathcal{F}_{\lambda}=\mathcal{F}_{\lambda}(\boldsymbol{\Omega}, t)$, where $\boldsymbol{\Omega}$ is the direction to the observer. To integrate the flux we need the specific intensity at each point of the surface at each time in the direction $\Omega$. In general, the specific intensity is a function of wavelength $\lambda$, the spatial point $\boldsymbol{r}$, the direction $\boldsymbol{\Omega}$ and the time. We want to restrict this to a simpler situation, where the intensity can be calculated from a plane-parallel model atmosphere at each point. Furthermore we assume, that the surface structure is equivalent to a static model atmosphere down to optical depths larger than 1 for each time step. In this case the specific intensity is only a function of the wavelength, the local flux $F$ and the cosine between $\Omega$ and the vector normal to the surface $\boldsymbol{n}$

$I_{\lambda}=I_{\lambda}(F, \mu)$,

with $\mu=\boldsymbol{n} \cdot \boldsymbol{\Omega}$. In this approximation the dependence of the specific intensity on the surface element specified
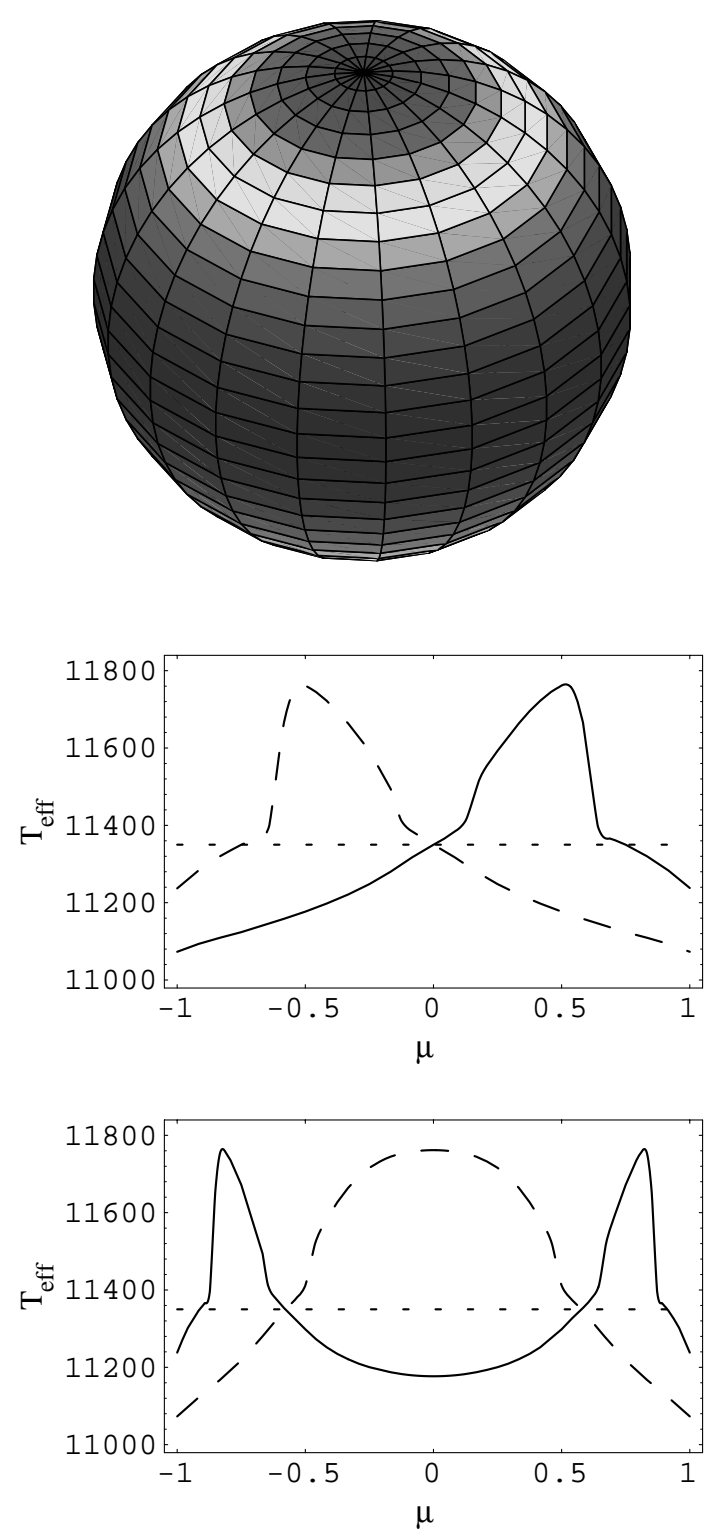

Fig. 8. Similar to Fig. 6, but for a maximum pressure amplitude of $20 \%$.

by the angles $\theta$ and $\phi$ and the time is not explicit but arises from the the dependence of the local flux on these quantities.

The surface coordinates $(\theta, \phi)$ can be taken as identical to the coordinates in the spherical harmonic of the pulsation mode. For the integrated flux it is convenient to introduce a coordinate system with the axis oriented towards the observer. The system $(\vartheta, \varphi)$ is defined by

$\vartheta=0 \quad$ for $\quad \mu=1$.

In this case the integrated flux can be written as

$\mathcal{F}_{\lambda}(\boldsymbol{\Omega}, t)=\int_{0}^{2 \pi} \mathrm{d} \varphi \int_{0}^{1} \mathrm{~d} \cos \vartheta \cos \vartheta I_{\lambda}(F(\vartheta, \varphi, t), \mu)$. 
Assuming that the flux $\mathcal{F}_{\lambda}$ is strictly a periodic function with the circular frequency $\omega$ we can expand the flux in a Fourier series

$\mathcal{F}_{\lambda}(\Omega, t)=\frac{A_{0}}{2}+\sum_{n=1}^{\infty}\left[A_{n} \cos n \omega t+B_{n} \sin n \omega t\right]$

with

$A_{n}=\frac{\omega}{\pi} \int_{t_{0}}^{t_{\mathrm{o}}+2 \pi / \omega} \mathrm{d} t \mathcal{F}_{\lambda}(\Omega, t) \cos n \omega t$

and

$B_{n}=\frac{\omega}{\pi} \int_{t_{0}}^{t_{0}+2 \pi / \omega} \mathrm{d} t \mathcal{F}_{\lambda}(\Omega, t) \sin n \omega t$.

$A_{0} / 2$ is the mean surface-integrated flux. The amplitude for each harmonic $n$ is defined by

$\frac{\delta \mathcal{F}}{\mathcal{F}}_{n} \equiv \frac{2 \sqrt{A_{n}^{2}+B_{n}^{2}}}{A_{0}}$

and the normalized amplitude spectrum by

$\mathcal{Q}_{n} \equiv \frac{\frac{\delta \mathcal{F}}{\mathcal{F}}{ }_{n}(\lambda)}{\frac{\delta \mathcal{F}}{\mathcal{F}}{ }_{n}\left(\lambda_{0}\right)}=\frac{A_{0}\left(\lambda_{0}\right)}{A_{0}(\lambda)} \frac{\sqrt{A_{n}^{2}(\lambda)+B_{n}^{2}(\lambda)}}{\sqrt{A_{n}^{2}\left(\lambda_{0}\right)+B_{n}^{2}\left(\lambda_{0}\right)}}$.

Here $\lambda_{0}$ is an arbitrary reference wavelength, often taken in the visual at $5500 \AA$ corresponding to the $V$ magnitude.

\subsection{Relation of $I_{\lambda}$ to the local flux $F$}

For the evaluation of Eq. (11) it is necessary to connect the specific intensity with the total flux of a local column. Using an expansion to first order we assume for this relation

$I_{\lambda}=I_{\lambda}\left(F_{0}, \mu\right)-\left.\frac{\partial I_{\lambda}}{\partial F}\right|_{F_{0}}(\mu) F_{0}+\left.\frac{\partial I_{\lambda}}{\partial F}\right|_{F_{0}}(\mu) F(t)$.

Expanding the local flux in a Fourier series in the same manner as for the surface integrated flux

$F(t)=\frac{a_{0}}{2}+\sum_{n=1}^{\infty}\left[a_{n} \cos n \omega t+b_{n} \sin n \omega t\right]$

the amplitude for the harmonic $n$ is

$\frac{\delta F}{F}_{n} \equiv \frac{2 \sqrt{a_{n}^{2}+b_{n}^{2}}}{a_{0}}$

Inserting (16) into (11) one obtains after some manipulation (Wu 1998) for $n>0$ the very simple result

$A_{n}=\left.\int_{0}^{2 \pi} \mathrm{d} \varphi \int_{0}^{1} \mathrm{~d} \cos \vartheta \cos \vartheta \frac{\partial I_{\lambda}}{\partial F}\right|_{F_{0}}(\mu(\vartheta, \varphi)) a_{n}$
$B_{n}=\left.\int_{0}^{2 \pi} \mathrm{d} \varphi \int_{0}^{1} \mathrm{~d} \cos \vartheta \cos \vartheta \frac{\partial I_{\lambda}}{\partial F}\right|_{F_{0}}(\mu(\vartheta, \varphi)) b_{n}$.

The integration for $A_{0}$ leads to two additional terms and reduces to the simple form above only if the local timeaveraged flux $a_{0} / 2$ is equal to the equilibrium flux $F_{0}$ for the local column.

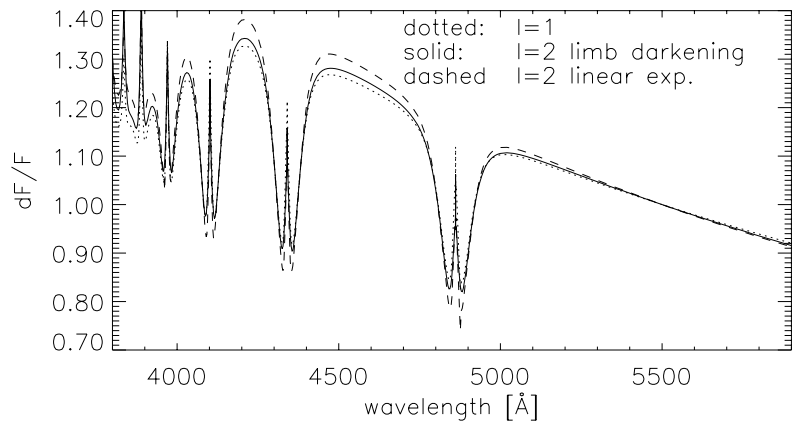

Fig. 9. Comparison of the amplitude spectra for an $l=2$ mode, calculated with a limb darkening independent of $T_{\text {eff }}$ (solid line) and with the linear expansion of the intensity (dashed line). The spectra for the $l=1$ mode are very similar and represented by the dotted line in the plot.

Using approximations similar to those of Robinson et al. (1982) we could simplify the relation for the intensity as

$I_{\lambda}=h_{\lambda}(\mu) F$

with a limb darkening function $h_{\lambda}$. The major simplification is that in this form the limb darkening does not depend on the total flux and the intensity derivative in Eq. (16) is simply given by $h_{\lambda}$. In this case the integral for the global coefficient $A_{0}$ always reduces to the simple form of Eq. (18). However, we will show below that this approximation is not adequate, since the the amplitude spectra depend very sensitively on the limb darkening and in the derivative of Eq. (20)

$\left.\frac{\partial I_{\lambda}}{\partial F}\right|_{F_{0}}=h_{\lambda}\left(\mu, F_{0}\right)+\left.\frac{\partial h_{\lambda}}{\partial F}\right|_{F_{0}}$

the second term is in general not negligible.

\subsection{The linear assumption}

The linear assumption is to specify the local flux by

$\frac{\delta F}{F}=\frac{\delta F}{F}_{1}^{\max } Y_{l}^{m}(\theta, \phi) \mathrm{e}^{i \omega t}$.

If we want to determine the global flux amplitudes directly from the local amplitudes without the intermediate step of Fourier coefficients we need to make further assumptions. If the coefficient $a_{0}$ and the ratio $a_{n} / b_{n}$ are constant over the stellar surface, it is possible to change the order of the square root and the integration over the visible disk in Eqs. (14), (18), (19). After some algebraic manipulations we get

$\frac{\delta \mathcal{F}}{\mathcal{F}}{ }_{n}=\frac{\left.\int_{0}^{2 \pi} \mathrm{d} \varphi \int_{0}^{1} \mathrm{~d} \cos \vartheta \cos \vartheta \frac{\partial I_{\lambda}}{\partial F}\right|_{F_{0}}(\mu(\vartheta, \varphi)) \frac{\delta F}{F} n}{\left.\int_{0}^{2 \pi} \mathrm{d} \varphi \int_{0}^{1} \mathrm{~d} \cos \vartheta \cos \vartheta \frac{\partial I_{\lambda}}{\partial F}\right|_{F_{0}}(\mu(\vartheta, \varphi))}$

It is common to transform the spherical harmonic $Y_{l}^{m}(\theta, \phi)$ to a system of spherical harmonics in the coordinate system $(\vartheta, \varphi)$ by

$Y_{l}^{m}(\theta, \phi)=\sum_{-l \leq m^{\prime} \leq l} R_{m^{\prime} m}^{l} Y_{l}^{m^{\prime}}(\vartheta, \varphi)$. 
As was shown in several papers (e.g. Robinson et al. 1982) all terms with $m^{\prime} \neq 0$ do not contribute to a flux variation in the integrated light, because they are equivalent to a rotating pattern with respect to an axis directed to the observer. For the term with $m^{\prime}=0$ the assumption of a constant phase over the stellar surface is valid and the Fourier coefficient $a_{0}$ is assumed to be constant over the surface by Eq. (22) implicitly. Consequently, we can use Eq. (23) to calculate the amplitude of the integrated flux with the local amplitude

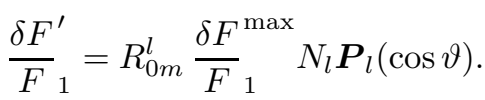

The quantity $N_{l}$ symbolizes the normalization of the spherical harmonic $Y_{l}^{0}$ and $\boldsymbol{P}_{l}$ is the Legendre polynomial. The only inclination dependent term in Eq. (24) is the coefficient $R_{0 m}^{l}$. The amplitude $(\delta F / F)_{1}^{\max }$ and $R_{0 m}^{l}$ are constant over the whole surface and wavelength independent. Consequently, the normalized spectra $\mathcal{Q}$ are independent of amplitude and inclination. Figure 9 shows one example of the results for a flux independent limb darkening and for the more general form of the linearized intensity for the model parameters we use in this paper, but with the linear assumption of Eq. (22). Both results are identical for an $l=1$ mode, but differ for larger $l$. Even for the $l=2$ modes, there are significant differences.

To test the accuracy of the linear approximation for the intensity (Eq. (16)) we have calculated the Fourier coefficients directly from the integrated light curve by means of Eqs. (11), (12) and (13) and have compared the result with the results from the linearized intensity. We find a very good correspondence for all wavelengths for modes with $l \leq 3$ and amplitudes less than $30 \%$. This range is much larger than the range, where the total flux variation can be assumed to be linear with $T_{\text {eff }}$. For even larger amplitudes the amplitude spectrum calculated directly from Eq. (11) becomes inclination and amplitude dependent, because the function $h_{\lambda}$ varies over the surface for different flux amplitudes. Such large amplitudes are not observed, however.

\subsection{The Goldreich and Wu theory}

The Goldreich and Wu theory assumes - following Brickhill - a pressure variation with a spatial and timedependence described by the spherical harmonic and period of the pulsation mode. They predict the appearance of non-sinusoidal flux variations at the surface

$\frac{\delta F}{F}=\frac{a_{0}}{2}+\frac{\delta F}{F_{1}} \sin \left(\omega t-\alpha_{1}\right)+\frac{\delta F}{F_{2}} \sin \left(2 \omega t-\alpha_{2}\right)+\ldots$

An important result of their calculation is that the amplitude of the fundamental is proportional to the pressure amplitude and the spatial distribution therefore still given by the same spherical harmonic. The first overtone varies quadratically with the pressure, which leads to the appearance of a limited number of spherical harmonics.
The phases $\alpha_{n}$ are independent of the amplitude of the variation.

The result for the fundamental is equivalent to the linear assumption Eq. (22) and as a consequence leads to the same results for the normalized spectra: they depend only on the mode number $l$.

The amplitude of the first overtone has a different behavior than the spherical harmonic of the mode over the stellar surface. It can be written as

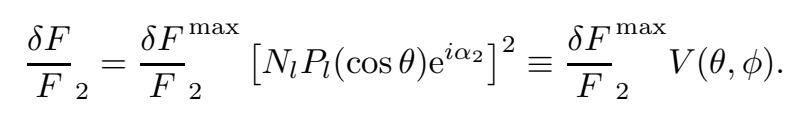

The angle dependent function $V$ can be expanded in a series of spherical harmonics

$V(\theta, \phi)=\sum_{l^{\prime}=0}^{\infty} \sum_{m^{\prime}=-l^{\prime}}^{l^{\prime}} c_{l^{\prime}}^{m^{\prime}} Y_{l^{\prime}}^{m^{\prime}}(\theta, \phi)$

It is obvious that in this case $V$ is a polynomial of the order $2 l$ and consequently all coefficients $c_{l^{\prime}}^{m^{\prime}}$ vanish for $l^{\prime}>2 l$, but for this discussion we only need, that the sum has in general more than one term. Analogous to the linear assumption we can transform each term of Eq. (27) to the coordinate system of the observer

$$
V(\theta, \phi)=\sum_{l=0}^{\infty} \sum_{m^{\prime}=-l^{\prime}}^{l^{\prime}} \sum_{m^{\prime \prime}=-l^{\prime}}^{l^{\prime}} c_{l^{\prime}}^{m^{\prime}} R_{m^{\prime \prime} m^{\prime}}^{l^{\prime}} Y_{l^{\prime}}^{m^{\prime \prime}}(\vartheta, \varphi) .
$$

The relation Eq. (28) can be introduced in Eq. (26) and we get an expression for $(\delta F / F)_{2}$ in Eq. (23). Again, only the terms with $m^{\prime \prime}=0$ lead to a non-vanishing amplitude in the integrated light. Inclination dependent quantities are only the coefficients $R_{0 m^{\prime}}^{l^{\prime}}$, but they are different for all $l^{\prime}$. Consequently, the inclination dependence does not cancel in the normalized spectrum $\mathcal{Q}_{2}$ and the spectrum becomes inclination dependent. In contrast to this, the amplitude $(\delta F / F)_{2}^{\max }$ cancels out, and the normalized amplitude spectrum remains independent of the amplitude.

The $2^{\text {nd }}$ overtone will not be discussed in detail. The local flux amplitude $(\delta F / F)_{3}$ cannot be represented by a single $(\delta F / F)^{\max }$ and consequently the normalized amplitude spectra become in general also amplitude dependent.

\section{Amplitude spectra from numerical calculations}

To determine the normalized amplitude spectra from our numerical results, it is possible to use the integration of the local Fourier coefficients $a_{n}$ and $b_{n}$. However, because the phase is not constant (see Fig. 4) over the surface and $a_{0}$ is a function of the coordinates $(\theta, \phi)$ (see Fig. 5) the expression Eq. (23) is completely useless even for $m=$ 0 modes and we have to calculate the coefficients $A_{n}$ and $B_{n}$ separately. This means that there is no economical advantage to calculate the Fourier series locally and then to integrate the coefficients over the visible disk.

We therefore decided to calculate the flux $\mathcal{F}_{\lambda}$ for each time step completely numerically by using Eq. (11). This method also avoids the disadvantage of having to use a 


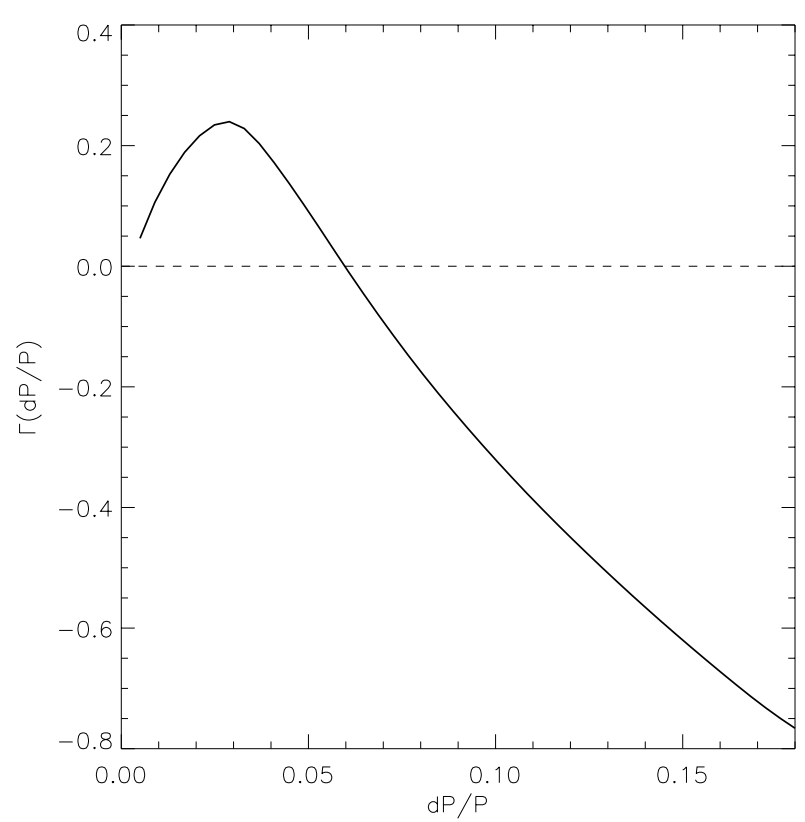

Fig. 10. The amplitude dependence of the correction term $\mathcal{Q}_{n}^{\text {corr }}$. The factor $\Gamma$ is displayed for our model parameters.

linearization like Eq. (16) to reach expressions for the Fourier coefficients of the integrated flux $\mathcal{F}_{\lambda}$. From the time-dependent flux we can calculate the Fourier coefficient with the aid of Eqs. (12) and (13). The numerical results presented in the following section are based on such integrations.

On the other hand, in order to gain more insight into the qualitative behavior of the spectra for different amplitudes and inclinations, we will use simple fits for the numerical results of the local variation, which allow then analytic integrations for the total flux. Both approaches are complementary: with the completely numerical simulation we avoid simplifications, which may not be correct, but we can calculate the result only for very few combinations of parameters. The semi-analytical study, on the other hand, shows the functional dependence of the results on quantum numbers, inclination, etc.

\subsection{General effects on the spectra}

The phase dependence of all local Fourier coefficients and the nonlinear dependence on the pressure variation leads to deviations from the assumptions made traditionally for the flux variation over the stellar surface. The intention of this subsection is to show, how this deviation affects the synthetic spectra for different inclinations, pulsation amplitudes and mode numbers $l$.

To find an expression for the error made when using the linear assumption, we assume a flux independent limb darkening function and the integrated Fourier coefficients given by Eqs. (18) and (19). We define a correction term for the fundamental as

$\mathcal{Q}_{n}^{\text {corr }} \equiv \frac{\frac{\delta \mathcal{F}}{\mathcal{F}_{n}}(\lambda)}{\frac{\delta \mathcal{F}}{\mathcal{F}}{ }_{n}\left(\lambda_{0}\right)}-\left.\frac{\frac{\delta \mathcal{F}}{\mathcal{F}}{ }_{n}(\lambda)}{\frac{\delta \mathcal{F}}{\mathcal{F}}{ }_{n}\left(\lambda_{0}\right)}\right|_{\text {linear }}$

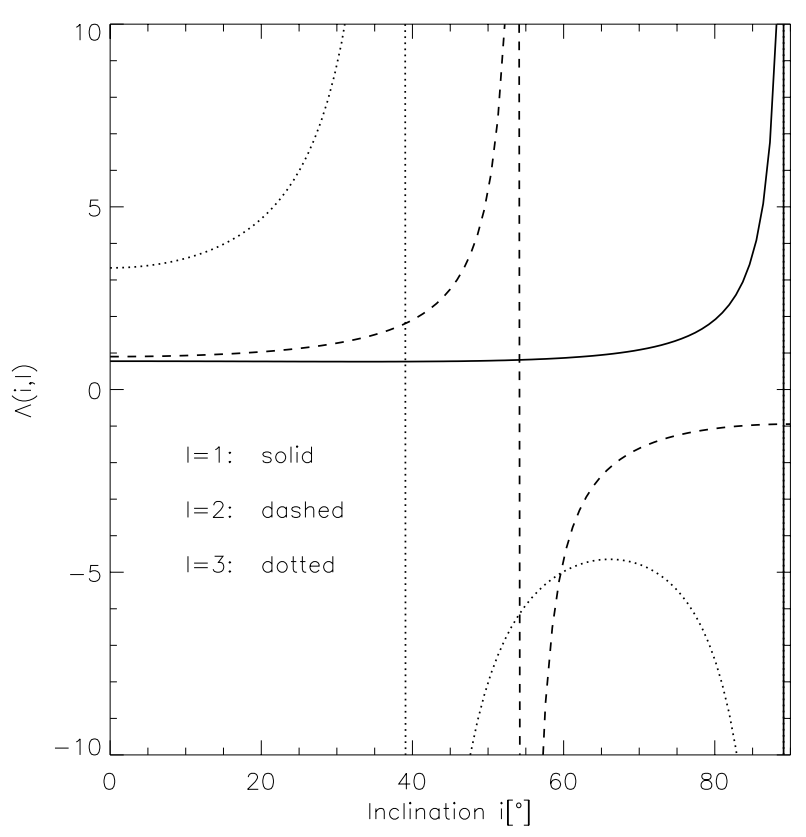

Fig. 11. The factor $\Lambda$ is shows the dependence of the correction factor between the linear assumption and the numerical simulation on inclination. The 3 curves show the factor for 3 different mode numbers $(l=1,2,3)$ and the Edington limb darkening law. Large deviations occur near an inclination of 55 degrees.

In Appendix A we demonstrate that in a first order approximation $\mathcal{Q}_{1}^{\text {corr }}$ can be separated into three factors

$\mathcal{Q}_{1}^{\text {corr }}=\frac{A_{0}\left(\lambda_{0}\right) \mathcal{I}_{0}(\lambda)}{A_{0}(\lambda)} \Gamma\left(\frac{\delta P}{P}\right.$ max $) \Lambda(i, l)$.

The first term is practically independent of wavelength $\left(\mathcal{I}_{0}\right.$ is defined in Eq. (35) in the Appendix). The second term $\Gamma$ is derived from the expansion coefficients of the local flux as a function of the pressure variation. For finite amplitudes we interpret these coefficients as fit parameters to describe the local flux as a polynomial of 2nd order up to the maximum pressure variation, that occurs at the stellar surface. This is plotted in Fig. 10. $\Gamma$ is not a function of the inclination or the mode numbers, but depends on the stellar parameter as $T_{\text {eff }}$ and the pulsation period. For our model parameters we can divide the synthetic spectra in three different pressure regimes: small amplitudes up to $\delta P / P_{\max } \sim 5 \%$, where the function $\Gamma$ is positive; intermediate amplitudes $5 \%<\delta P / P_{\max }<7 \%$, where $\Gamma$ is approximately 0 , and large amplitudes $\delta P / P_{\max }>7 \%$ with a negative $\Gamma$. In the following 3 subsections these cases are discussed separately for the numerical results.

The third term $\Lambda$ is the inclination dependent term. Besides this it depends on the mode numbers and wavelength, but not on the amplitude. The results for the factor $\Lambda$ for $l \leq 3$ are plotted in Fig. 11. This factor is for most inclinations very small for $l=1$ and moderate for $l=2$, if the inclination is clearly different from the latitude of the node lines. For $l=3$ the factor $\Lambda$ is much larger and leads to a large global deviation of the numerical results from 


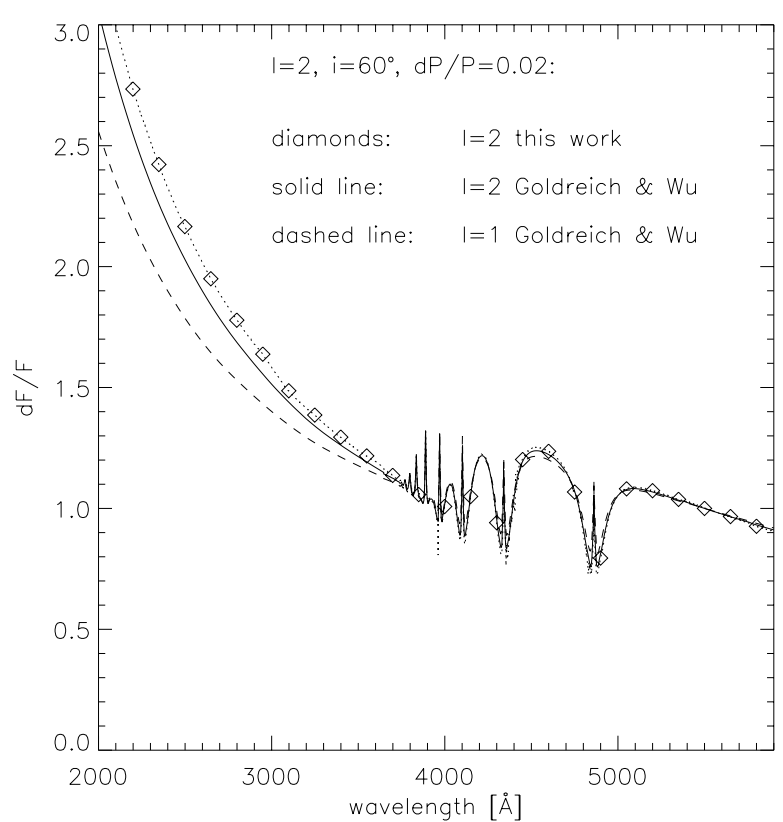

Fig. 12. Amplitude spectra for small pressure amplitude. The solid line and the dashed lines are results based on the spherical harmonics $l=2$ and $l=1$ respectively. The diamonds connected with the dotted line are the numerical results.

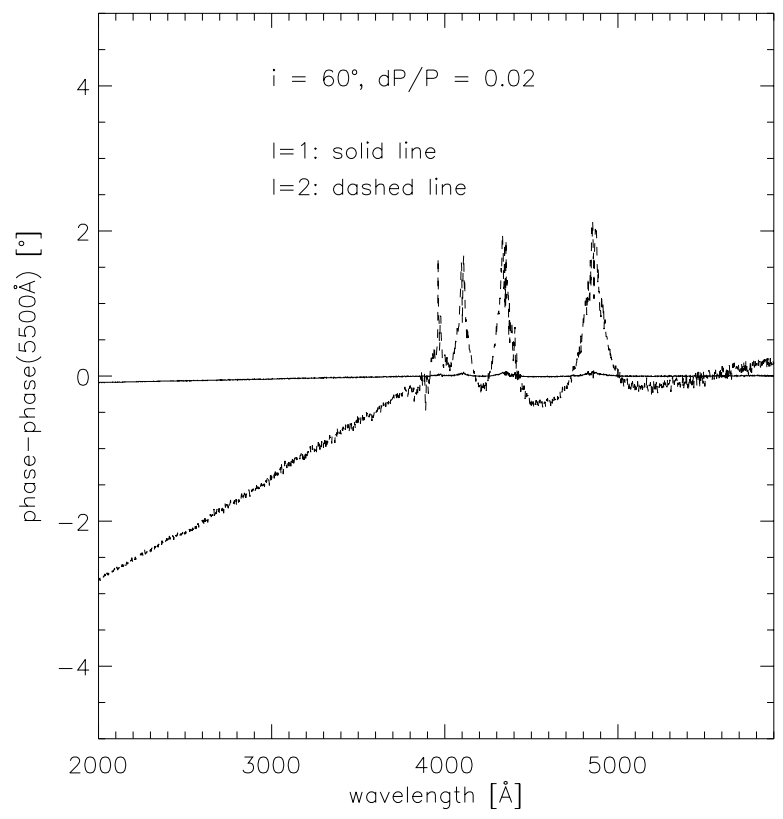

Fig. 13. Phase spectra for small pressure amplitude. The solid line $(l=1)$ and the dashed line $(l=2)$ gives the predicted phase shift for the wavelength range from $2000 \AA$ to $6000 \AA$.

those using the linear assumptions for any inclination and amplitude.

\subsubsection{Small amplitudes}

In the previous section we discussed the effects of the nonlinear behavior of the light curves on the amplitude spectra of the fundamental with some approximations. To find the correct spectra we now calculate the Fourier

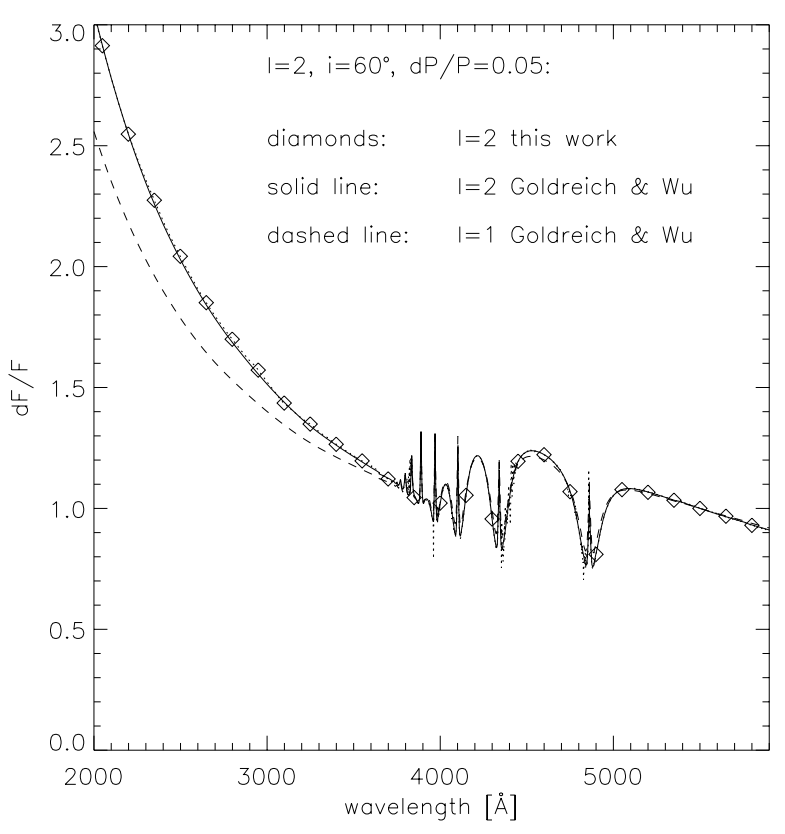

Fig. 14. Amplitude spectra for intermediate amplitude. The lines have the same meaning as in Fig. 12.

coefficients directly from the integrated light curves in a strictly numerical way.

As an illustrating example we take the $l=2$ mode with a maximum pressure amplitude of $2 \%$. For this amplitude the light curves are sinusoidal in a good approximation, so we can restrict the discussion to the fundamental of the mode.

From Fig. 11 we do not expect very large deviations from the linear result for most inclinations. To show the effect, we take an inclination of $60^{\circ}$ near the latitude of the node line, where we expect a moderate deviation. The numerical result is plotted in Fig. 12, together with the result of the linear assumption, that is supported by the theory of Goldreich and Wu. All amplitudes in this and the following similar figures have been normalized to 1 at $5500 \AA$. Although the effect is not very large, it is important for mode identification, because of the generally small differences between the calculations for different mode numbers.

Deviations in the spectra already for small amplitudes are suggested by the analytical calculations in the previous subsection, but are not obvious in the plot of the absolute amplitude Fig. 3, which grows linearly with the pressure amplitudes up to $\sim 5 \%$ pressure amplitude. Consequently, the deviation has to be an effect of the phase shift for small amplitudes. This phase shift should be visible in the integrated light as well. Figure 13 gives the numerical prediction for the phase shift for the same mode and modes with $l=1$. In contrast to the prediction of Goldreich and $\mathrm{Wu}$, we expect a small phase shift of a few degrees over the spectral range for the $l=2$ modes.

\subsubsection{Intermediate amplitudes}

The amplitude dependent factor $\Gamma$ is very small in this region and we expect practically no differences to the 


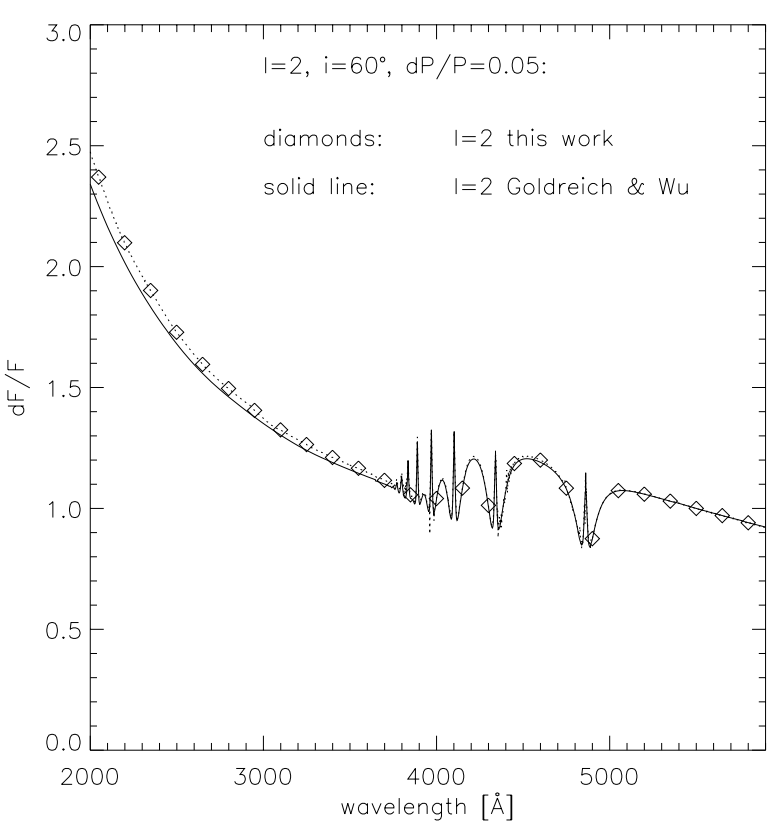

Fig. 15. Amplitude spectra for the first overtone and intermediate amplitude. The lines have the same meaning as in Fig. 12.

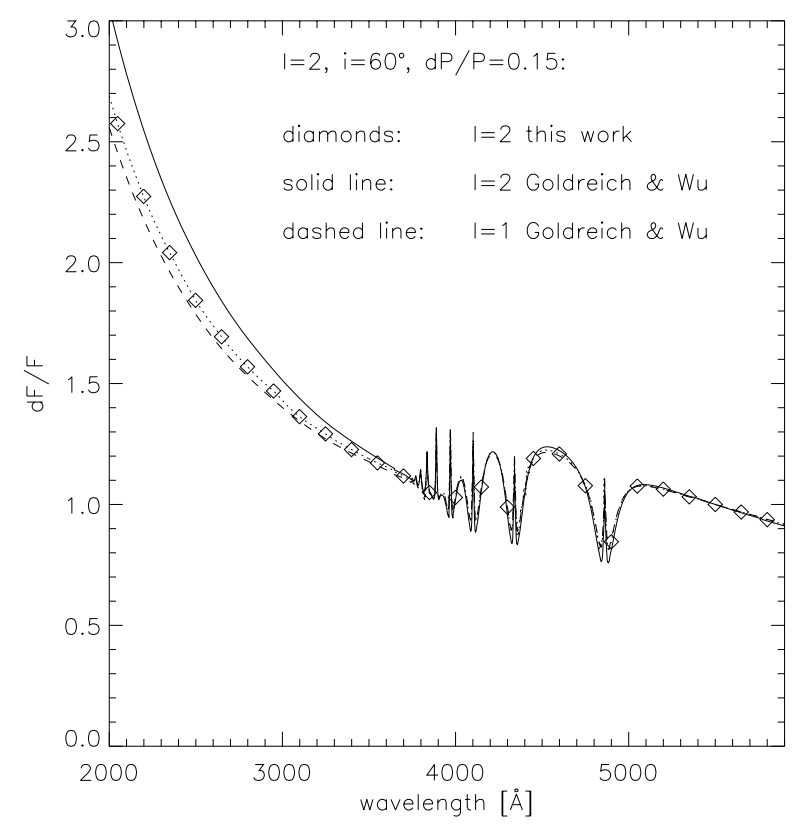

Fig. 16. Amplitude spectra for large amplitude. The lines have the same meaning as in Fig. 12.

predictions of Goldreich and Wu. Figure 14 shows the numerical result for the same mode and inclination as Fig. 12, but for a pressure amplitude of $5 \%$. The spectra for the numerical prediction and the Goldreich and $\mathrm{Wu}$ theory are very similar.

In contrast to the small amplitude case, the light curves for $5 \%$ pressure amplitude are non-sinusoidal with large flux amplitudes. We can calculate the expected deviation from the predictions of Goldreich and $\mathrm{Wu}$ analogous to Eq. (30) and find for this region a small deviation for the first overtone as well. Figure 15 shows a comparison of the numerical result, with the predicted spectrum for a

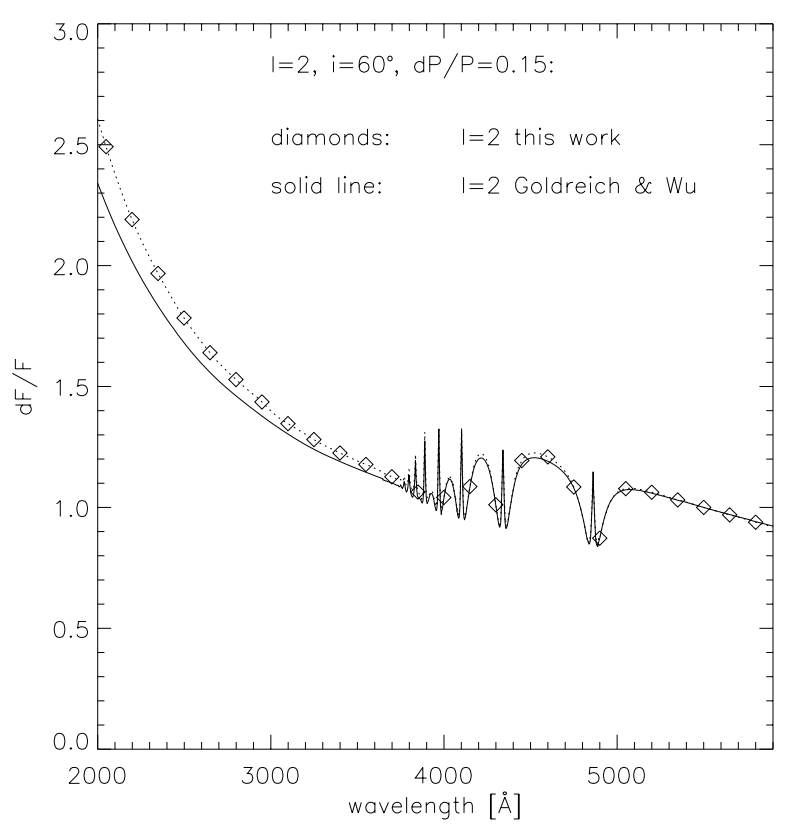

Fig. 17. Amplitude spectra for the first overtone and large amplitudes. The lines have the same meaning as in Fig. 12.

quadratic dependence of the flux amplitude on the pressure amplitude.

\subsubsection{Large amplitudes}

For increasing maximum pressure amplitude the deviation factor $\Gamma$ becomes smaller and finally negative. For really large pressure amplitude we expect again a significant deviation from the Goldreich and Wu result. Figure 16 shows the numerical result for a $15 \%$ pressure amplitude. As expected from the analytical result, the difference to the Goldreich and $\mathrm{Wu}$ result has the opposite sign and the spectrum of the $l=2$ mode becomes very similar to that of an $l=1$ mode. The numerical result for the $l=1$ mode does not significantly change for an inclination of $60^{\circ}$ (see Fig. 11) and modes with $l=2$ can easily be confused with $l=1$.

The first overtone is significant for large amplitudes as well. In contrast to the intermediate amplitudes, a difference from the predicted result of Goldreich and $\mathrm{Wu}$ appears. For our example Fig. 17 shows the numerical result in comparison with the quadratic dependence of the flux amplitude.

For $l=3$ the deviation of the numerical results from the results of the linear assumption is much larger. Figure 18 shows one example for a large deviation. In contrast to a rapidly increasing amplitude in the UV the amplitude remains almost constant. In this regime the prediction of the variation from Eq. (30) can be only qualitative. In general, the amplitude increase towards the UV is caused by the decreasing "effective visible area" due to the larger limb darkening. This effect becomes significant, when the effective area is comparable in size to the spatial scale of the light variations on the surface of the star. For 


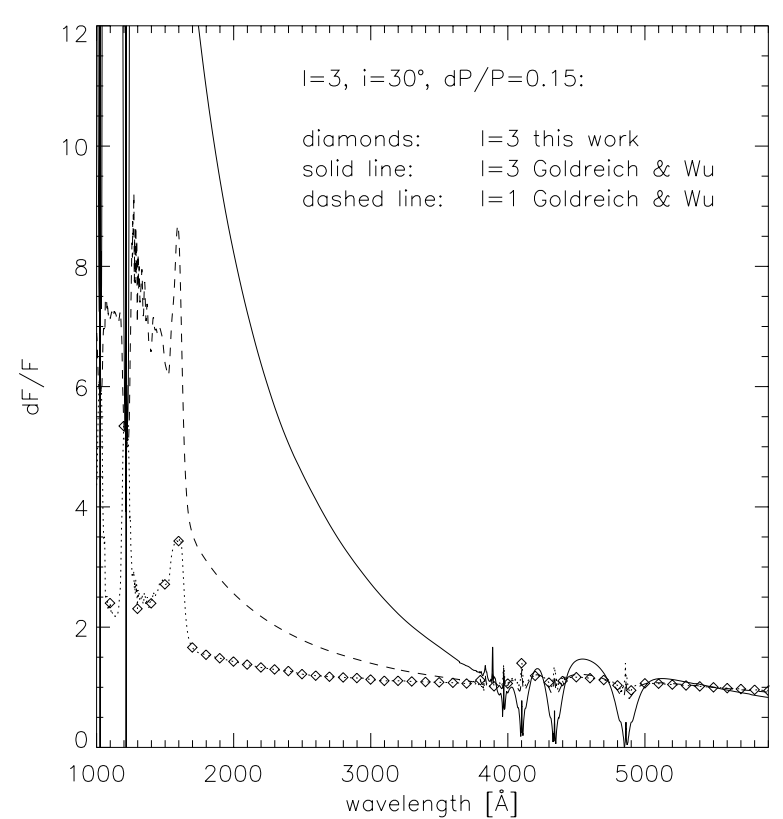

Fig. 18. Amplitude spectra for $l=3$ and large amplitudes. The lines have the same meaning as in Fig. 12.

the large amplitudes the spatial structure of the $l=3$ flux variation is more complex than the unperturbed spherical harmonic and the cancellation effects set in only farther in the UV.

\section{Conclusions}

In the past, the method of time-resolved spectroscopy has been based on the assumption - called "linear" throughout this paper - that the flux varies with the spherical harmonic of the pulsation mode over the surface of the ZZ Ceti stars. The numerical light curve simulation shows that this assumption is questionable in many situations. For small amplitudes with sinusoidal lightcurves, only the absolute amplitude varies like the spherical harmonic of the mode. The phase shift leads to normalized amplitude spectra, which depend on inclination and pulsation (pressure) amplitude for mode numbers $l>2$. For large amplitudes $(>10 \%$ in pressure) the absolute amplitude of the flux depends non-monotonously on the pressure amplitude and the flux variation shows maxima at different latitudes than the spherical harmonic of the mode. The numerical results show relatively good correspondence to traditionally calculated amplitude spectra for intermediate amplitudes $(\sim 5 \%)$ in pressure, with non-sinusoidal light curves. In this regime, the phase becomes stationary and the absolute amplitude varies in good correspondence to the spherical harmonic of the mode; the numerical results are close to the results of the perturbation analysis of Goldreich and Wu.

Another result of our simulations is the existence of a maximum surface flux amplitude. This is a reaction of the convection zone on a predefined pressure variation in deeper layers, and should not be confused with the amplitude saturation of the pulsation as studied by
Goldreich \& Wu (1999). The amplitude of the photospheric flux is reduced by the inert reaction of the temperature structure in the convective layer to the changing input flux. The decrease in the thermal heat flux is converted to kinetic energy of the pulsation as discussed above, and this convection region is therefore a driving region for the pulsation. The extent of this conversion depends on the thermal time scale of the convection zone.

In our model calculations for large amplitudes the time-averaged depth of the convection zone becomes much larger than in the corresponding static model. The thermal time scale then increases with the pulsation amplitude, leading to a reduction of the surface flux amplitude. This is the reason for the existence of a maximum amplitude for the surface flux. It also explains the return to sinusoidal variations for large pressure amplitudes: the convection zone becomes so thick that variations during one cycle do not decrease its depth enough for the appearance of higher overtones.

While our calculations cannot determine the maximum pressure amplitude that can be reached in a pulsating star, one prediction is that observable flux amplitudes should not exceed $10 \%$, for the parameters used in this paper. The maximum flux amplitudes support the dominance of small $l$ in the observed light curves and lead to the prediction that the dominant pulsation modes are $l=1$, if $l=1$ modes and modes with larger $l$ are unstable, for a large amplitude range, independent of the actual amplitudes of the modes.

While nonlinear effects are well known for large observed amplitude pulsations, the present study reaches the surprising conclusion that the amplitudes and phases can be described fairly accurately by the linear theory as developed by Robinson et al. (1982) for the fundamental and $\mathrm{Wu}(1998)$ for higher harmonics.

On the other hand, the observation of small amplitudes in the surface integrated light does not necessarily guarantee that these effects are absent. Deviations from the traditional interpretation could occur in spite of small observed amplitudes under the following conditions

- the pressure amplitude is larger than about $12 \%$, that is, in the range were the flux amplitude decreases again with increasing pressure amplitude;

- the flux variations are intrinsically large on the surface but the inclination is close to the latitude of a node line;

- the mode number $l$ is large.

In all these cases, one of the correction factors describing the difference between our numerical results and the amplitudes calculated with the linear assumptions becomes large, and mode identification from time-resolved spectroscopy will only be possible with extended numerical simulations.

All conclusions are obtained for a special set of stellar and pulsational parameters. The effects discussed may be more or less important for other ranges of $\log g$ and $T_{\text {eff }}$. We have also not taken into account that in general more 
than one pulsation mode is present in a star. The modes will be influenced by the presence of other modes and by their properties even well below the convection zone. These effects are beyond the scope of the present analysis. We do not, however, expect that the main conclusion will change: the mechanism that leads to non-sinusoidal light curves for large flux variations can be important for a correct identification of the pulsation modes using timeresolved spectroscopy, for any observed amplitude of the flux.

Acknowledgements. This work was made possible by a grant from the Deutsche Forschungsgemeinschaft. We want to thank Dr. Yanqin Wu, Dr. Marten van Kerkwijk, Prof. Dr. Rainer Wehrse and Dr. Günther Wuchterl for their helpful comments and recommendations. D.K. wants to thank Dr. J. Holberg of Lunar and Planetary Lab and Dr. J. Liebert of Steward Observatory for their hospitality during a stay at the University of Arizona in the summer of 2000 and the DFG for financial support.

\section{Appendix A: Difference between the linear spectra and the numerical results}

To find a semi-analytic expression for the difference between the synthetic amplitude spectra from the linear theory and our numerical calculation, we approximate the local Fourier coefficients $a_{1}$ and $b_{1}$ as a quadratic function of the local pressure amplitude. Assuming a pressure variation with the spherical harmonic of the mode on the stellar surface we can write for the modes with $l=0$

$a_{1} \approx \alpha_{1}\left(\frac{\delta P}{P}_{\text {max }}\right) \boldsymbol{P}_{l}+\alpha_{2}\left(\frac{\delta P}{P}_{\text {max }}\right)\left(\boldsymbol{P}_{l}\right)^{2}$.

The quatities $\alpha_{1}, \alpha_{2}$ are given by the fit to the numerical results and thus depend on the maximum pressure amplitude on the surface.

In a similar way as for the spherical harmonics we can also expend $\left(\boldsymbol{P}_{l}\right)^{2}$ with a finit number of $\boldsymbol{P}_{l^{\prime}}$. In this first order approximation we only need the term with $l^{\prime}=0$ of this expansion (the next higher term belongs to $l^{\prime}=2$ and is strongly reduced by the cancelation effect). We can write

$\left(\boldsymbol{P}_{l}\right)^{2} \approx \frac{\boldsymbol{P}_{0}}{2 l+1}=\frac{1}{2 l+1}$.

Now it is possible to perform the rotation to the coordinate system of the observer in the space of the spherical harmonic. The result is a mixing of all possible quantum numbers $m^{\prime}$ to the same $l$. As in the linear case we only have to consider the contribution of $m^{\prime}=0$ to the visible pulsation amplitude and get

$a_{1} \approx \alpha_{1} \boldsymbol{P}(\cos i) \boldsymbol{P}(\cos \vartheta)+\alpha_{2} \frac{1}{2 l+1}$,

or after the integration over the visible disc

$A_{1} \approx \alpha_{1} \boldsymbol{P}(\cos i) \mathcal{I}_{l}\left(\lambda, F_{0}\right)+\alpha_{2} \frac{1}{2 l+1} \mathcal{I}_{0}\left(\lambda, F_{0}\right)$ with

$\left.\mathcal{I}_{l} \equiv 2 \pi \int_{0}^{1} \mathrm{~d} \cos \vartheta \cos \vartheta \frac{\partial I}{\partial F}\right|_{F_{0}}(\cos \vartheta) \boldsymbol{P}_{l}(\cos \vartheta)$.

To find the Fourier amplitude we insert this expression and the anlogous equation for $B_{1}$ in (14) and get for small $\alpha_{2}, \beta_{2}\left(\beta_{1}\right.$ and $\beta_{2}$ are the fit parameters for $\left.B_{1}\right)$

$$
\frac{\delta \mathcal{F}}{\mathcal{F}_{1}} \approx \frac{2}{A_{0}} \frac{\sqrt{\left(\alpha_{1}^{2}+\beta_{1}^{2}\right)\left(\boldsymbol{P}_{l}(\cos i) \mathcal{I}_{l}\right)^{2}} \cdots}{\cdots+\left(\alpha_{1} \alpha_{2}+\beta_{1} \beta_{2}\right) \frac{\boldsymbol{P}_{l}(\cos i) \mathcal{I}_{0} \mathcal{I}_{l}}{2 l+1}}
$$

or by expanding the sqare root

$$
\begin{aligned}
\frac{\delta \mathcal{F}}{\mathcal{F}_{1}} \approx \frac{2}{A_{0}} & \sqrt{\alpha_{1}^{2}+\beta_{1}^{2}} \boldsymbol{P}_{l}(\cos i) \mathcal{I}_{l} \\
& +\frac{2}{A_{0}} \frac{\alpha_{1} \alpha_{2}+\beta_{1} \beta_{2}}{(2 l+1) \sqrt{\alpha_{1}^{2}+\beta_{1}^{2}}} \mathcal{I}_{0} .
\end{aligned}
$$

The first term of this expression is proportional to the result of the linear theory with the assumption of a flux variation with a spherical harmonic. The second term is the first order nonlinear correction term for the numerical result. For this first order approximation it is sufficient to identify the mean flux $A_{0} / 2$ with the flux of the nonpulsating star $\mathcal{I}_{0}$. We get for the correction term of the amplitude

$\frac{\delta \mathcal{F}}{\mathcal{F}}_{1}^{\text {corr }} \approx \frac{\alpha_{1} \alpha_{2}+\beta_{1} \beta_{2}}{(2 l+1) \sqrt{\alpha_{1}^{2}+\beta_{1}^{2}}}$.

This expression is a function of $T_{\text {eff }}$ of the equilibrium model, the pulsation mode and the maximum pulsation amplitude of the model; it does not depend on the wavelength nor on the inclination. The linear term, however, which is normally dominant, is a very sensitive function of the inclination.

Following the standard strategy to find an expression, which eliminates the inclination dependence of the linear term, we normalize Eq. (37) to the amplitude at a reference wavelength $\lambda_{0}$ (e.g. $\left.=5500 \AA\right)$ and obtain for the normalized spectrum

$\mathcal{Q}_{1} \approx \mathcal{Q}_{1}^{\text {lin }}+\mathcal{Q}_{1}^{\text {corr }}$

with the linear normalized spectrum $\mathcal{Q}_{1}^{\operatorname{lin}}$ and

$\mathcal{Q}_{1}^{\text {corr }} \equiv \frac{A_{0}\left(\lambda_{0}\right) \mathcal{I}_{0}(\lambda)}{A_{0}(\lambda)} \Gamma\left(\frac{\delta P}{P}\right) \Lambda(i, l)$.

The inclination dependent term $\Lambda(i, l)$ is given by

$\Lambda(i, l) \equiv \frac{1}{2 l+1} \frac{1}{\boldsymbol{P}_{l}(\cos i) \mathcal{I}_{l}\left(\lambda_{0}\right)}$

and the amplitude dependent term by

$\Gamma\left(\frac{\delta P}{P}\right) \equiv \frac{\alpha_{1} \alpha_{2}+\beta_{1} \beta_{2}}{\alpha_{1}^{2}+\beta_{1}^{2}}$. 


\section{References}

Bergeron, P., et al. 1995, ApJ, 449, 258

Brassard, P., Fontaine, G., \& Wesemael, F. 1995, ApJS, 96, 545

Brickhill, A. J. 1983, MNRAS, 204, 537

Brickhill, A. J. 1990, MNRAS, 246, 510

Brickhill, A. J. 1991, MNRAS, 251, 673

Brickhill, A. J. 1991a, MNRAS, 252, 334

Brickhill, A. J. 1992, MNRAS, 259, 519

Brickhill, A. J. 1992a, MNRAS, 259, 529

Clemens, J. C. 1993, Baltic Astron., 2, 407

Clemens, J. C., van Kerkwijk, M. H., Wu, Y., \& Kleinman, S. J. 1999, ASP Conf. Ser., 169, 122

Clemens, J. C., van Kerkwijk, M. H., \& Wu, Y. 2000, MNRAS, 314,220

Cox, J. P. 1980, Theory of Stellar Pulsation (Princeton University Press)

Finley, D. S., Koester, D., \& Basri, G. 1997, ApJ, 488, 375
Gear, C. W. 1971, Numerical initial value problems in ordinary differential equations (Prentice-Hall)

Goldreich, P., \& Wu, Y. 1999, ApJ, 511, 904

Goldreich, P, \& Wu, Y. 1999a, ApJ, 523, 805

Iglesias, C. A., \& Rogers, F. J. 1996, ApJ, 464, 943

Kepler, S. O., Robinson, E. L., Koester, D., et al. 2000, ApJ, 539, 379

Kuhfuss, R. 1986, A\&A, 160, 116

Robinson, E. L., Kepler, S. O., \& Nather, R. E. 1982 ApJ, 259, 219

Robinson, E. L., Mailloux, T. M., Zhang, E., et al. 1995, ApJ, 438, 908

Saumon, D., Chabrier, G., \& Van Horn, H. M. 1995, ApJS, 99, 713

Wu, Y. 1998, Ph.D. Thesis

Wu, Y., \& Goldreich, P. 1999, ApJ, 519, 783

Wu, Y. 2000, submitted to MNRAS

Wuchterl, G., \& Feuchtinger, M. U. 1998, A\&A, 340, 419 